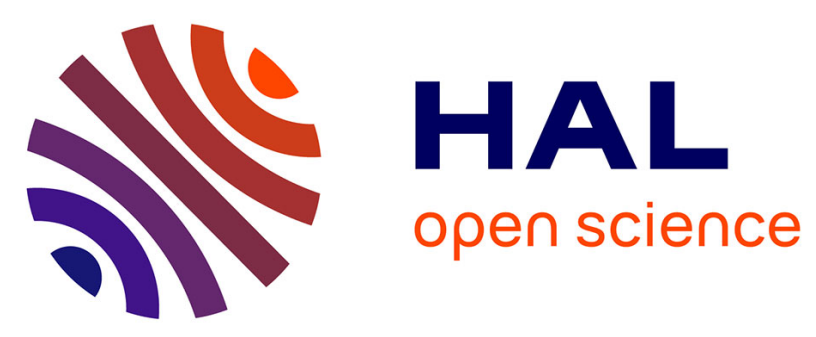

\title{
Wrapping Transition and Wrapping-Mediated Interactions for Discrete Binding along an Elastic Filament: An Exact Solution
}

David S. Dean, Thomas C. Hammant, Ronald R. Horgan, Ali Naji, Rudolf Podgornik

\section{To cite this version:}

David S. Dean, Thomas C. Hammant, Ronald R. Horgan, Ali Naji, Rudolf Podgornik. Wrapping Transition and Wrapping-Mediated Interactions for Discrete Binding along an Elastic Filament: An Exact Solution. Journal of Chemical Physics, 2012, 6 (10), pp.144904. 10.1063/1.4757392 . hal00758768

\section{HAL Id: hal-00758768 https://hal.science/hal-00758768}

Submitted on 28 Aug 2018

HAL is a multi-disciplinary open access archive for the deposit and dissemination of scientific research documents, whether they are published or not. The documents may come from teaching and research institutions in France or abroad, or from public or private research centers.
L'archive ouverte pluridisciplinaire HAL, est destinée au dépôt et à la diffusion de documents scientifiques de niveau recherche, publiés ou non, émanant des établissements d'enseignement et de recherche français ou étrangers, des laboratoires publics ou privés. 


\title{
Wrapping transition and wrapping-mediated interactions for discrete binding along an elastic filament: An exact solution
}

\author{
David S. Dean, ${ }^{1}$ Thomas C. Hammant, ${ }^{2}$ Ronald R. Horgan, ${ }^{2}$ Ali Naji, ${ }^{2,3, a)}$ \\ and Rudolf Podgornik ${ }^{4}$ \\ ${ }^{1}$ Université de Bordeaux and CNRS, Laboratoire Ondes et Matière d'Aquitaine (LOMA), UMR 5798, \\ F-33400 Talence, France \\ ${ }^{2}$ Department of Applied Mathematics and Theoretical Physics, University of Cambridge, \\ Centre for Mathematical Sciences, Cambridge CB3 OWA, United Kingdom \\ ${ }^{3}$ School of Physics, Institute for Research in Fundamental Sciences (IPM), Tehran 19395-5531, Iran \\ ${ }^{4}$ Department of Theoretical Physics, J. Stefan Institute, SI-1000 Ljubljana, Slovenia; Department of Physics, \\ Faculty of Mathematics and Physics, University of Ljubljana, SI-1000 Ljubljana, Slovenia; \\ and Department of Physics, University of Massachusetts, Amherst, Massachusetts 01003, USA
}

(Received 19 July 2012; accepted 19 September 2012; published online 10 October 2012)

\begin{abstract}
The wrapping equilibria of one and two adsorbing cylinders are studied along a semi-flexible filament (polymer) due to the interplay between elastic rigidity and short-range adhesive energy between the cylinder and the filament. We show that statistical mechanics of the system can be solved exactly using a path integral formalism which gives access to the full effect of thermal fluctuations, going thus beyond the usual Gaussian approximations which take into account only the contributions from the minimal energy configuration and small fluctuations about this minimal energy solution. We obtain the free energy of the wrapping-unwrapping transition of the filament around the cylinders as well as the effective interaction between two wrapped cylinders due to thermal fluctuations of the elastic filament. A change of entropy due to wrapping of the filament around the adsorbing cylinders as they move closer together is identified as an additional source of interactions between them. Such entropic wrapping effects should be distinguished from the usual entropic configuration effects in semi-flexible polymers. Our results may be relevant to the problem of adsorption of oriented nano-rods on semi-flexible polymers. () 2012 American Institute of Physics. [http://dx.doi.org/10.1063/1.4757392]
\end{abstract}

\section{INTRODUCTION}

Interaction, binding, and complexation to membranes and polymers, as typified by protein adsorption to DNA chains, play a vital role in biology and biochemistry. In biology, for example, a variety of proteins interact with DNA in cellular processes involving the reading and packaging of the genome. ${ }^{1}$ Transcription factors are proteins which bind to specific DNA sequences controlling the flow of genetic information to messenger RNA (mRNA) which in turn carries this information to ribosomes for protein synthesis. DNA polymerase is another type of protein (enzyme) that plays a key role in the DNA replication process: it synthesizes a new DNA strand by reading genetic information from an intact DNA strand that serves as a template. RNA polymerase is a different kind of DNA-binding protein that binds to DNA and uses it as a template to synthesize the RNA. In all these examples, protein binding induces local deformation of DNA. When multiple proteins bind to DNA they may exhibit a significant degree of cooperativity, ${ }^{2}$ which may result from the formation of loops, ${ }^{3}$ specific protein-protein interactions (at short separations), ${ }^{4}$ or a variable-range cooperative binding of proteins regulated by the tension along the DNA strand. ${ }^{5}$

Perhaps the most remarkable form of protein-induced deformation of DNA is found in the chromatin fibre. In eukary-

\footnotetext{
a)E-mail: a.naji@ipm.ir.
}

otic cells, a long strand of DNA is efficiently packed within a micron-size cell nucleus. This occurs through a hierarchical folding process ${ }^{1}$ where at the lowest length scale, short segments (about $50 \mathrm{~nm}$ ) of DNA are wrapped around small histone proteins forming nucleosomes: cylindrical wedgeshaped histone octamers of diameter of around $7 \mathrm{~nm}$ and mean height $5.5 \mathrm{~nm}$. DNA-histone binding may be mediated through specific binding sites ${ }^{6}$ as well as non-specific electrostatic interactions ${ }^{7-9}$ since both DNA and histones carry relatively large opposite (net) charges (on the average, around 188,130 , and 129 histone charges interact with the wrapped DNA for native chromatin, nucleosome core particle, and H1depleted chromatin, respectively, ${ }^{10}$ while DNA itself carries six elementary charges per $\mathrm{nm}$ ). In nucleosomes, DNA is strongly bent and wrapped around the core histones in nearly a 1-and-3/4 left-handed helical turn, which thus costs a large bending energy given that DNA has an effective persistence length of around $50 \mathrm{~nm}$ in physiological conditions. ${ }^{14}$

These DNA-histone complexes are linked together and, on the next level of the hierarchy, fold into a rather dense structure known as the $30 \mathrm{~nm}$ chromatin fibre, which undergoes a series of higher-order foldings resulting in highly condensed chromosomes. Under physiological conditions (salt concentration of around $100 \mathrm{mM} \mathrm{NaCl}$ ), this fibre exhibits a diameter of about $30 \mathrm{~nm}$, while at low salt concentrations the fibre is swollen and displays a beads-on-a-string pattern with a diameter of around $10 \mathrm{~nm}$ in which core particles are 
widely separated. ${ }^{6,15,16}$ In these cases, the wrapped structure of DNA around histone proteins remains intact. However, at salt concentrations below $1 \mathrm{mM}$, the DNA begins to partially unwrap from the histone cores. ${ }^{17}$ The electrostatic mechanism for the salt-induced wrapping-unwrapping behavior of the DNA around nucleosome core particles has been discussed in the literature for general classes of charged polymer-macroion complexes ${ }^{8,9,18-20}$ and for long complex fibres of multiple macroions ${ }^{21}$ and will not be considered in this paper. In general, the interplay between the DNA's bending energy cost, the electrostatic attraction energy as well as other specific binding energies would be the key to understand the global structural features of the chromatin fiber. The precise arrangement of nucleosome core particles in the $30 \mathrm{~nm}$ fibre is still intensely debated in the literature (see, e.g., Refs. 6, 15, 16, and 22-34 and references therein).

Genome-wide experimental mappings of nucleosome occupancy in yeast point to a patchy landscape composed of at least partly ordered crystal-like configurations with a nucleosome repeat length of about 165 base-pairs. ${ }^{11}$ It appears that what is encoded in the sequence of DNA is not the sequential ordering of nucleosomes but rather the nucleosome excluded regions. In between these regions nucleosomes position themselves via a thermodynamic equilibrium mechanism. ${ }^{12}$ The ordered configurations of nucleosomes between the excluded regions have been modeled as a nonuniform fluid of onedimensional (1D) hard rods confined by two excluding energy barriers at the extremities ${ }^{12,13}$ (see Ref. 21 for a ground-state treatment in three dimensions). The interaction between the nucleosomes was thus assumed to be of purely hard-core type, i.e., only steric exclusion is taken into account. Understanding the details of these interactions and the adsorption-wrapping equilibria for protein-DNA complexes is thus of paramount importance.

Motivated by these findings, we focus here on elastic properties and adsorption-desorption equilibria of a model system composed of adsorbing cylindrical particles and an elastic filament modeling a semi-flexible polymer chain with short-range adsorption interaction, assumed to be proportional to the arc-length of the filament touching the particle. We neglect any electrostatic interactions (which may be justified for charged species at high enough salt concentration in the solution) and focus on elasticity and short-range (adhesive) wrapping. Furthermore, we restrict ourselves to a twodimensional (2D) Eulerian plane model of a single elastic filament wrapped around one, two or many cylinders. This formulation of the model system allows us to introduce an $e x$ act formalism based on the Schrödinger representation for the partition function of the system and to carry out the calculations explicitly for the wrapping-unwrapping behavior of the elastic filament with one or two wrapping cylinders as well as for the effective interaction between two cylinders wrapped on a single filament. Our study may thus be relevant to the problem of adsorption of oriented nano-rods to semi-flexible polymers where off-plane deformations are negligible.

In the same context, the tension-mediated interaction between proteins bound to a DNA chain was initially considered by Rudnick and Bruinsma ${ }^{5}$ within the very same model used in the present paper. The thermal fluctuations of the chain were however dealt with only on a harmonic level, corresponding to small Gaussian fluctuations around the configuration of lowest energy. Our analysis, based on the Schrödinger representation, goes beyond this approximation and takes into account the whole spectrum of chain conformational fluctuations within a rigorous mathematical framework. In agreement with Ref. 5, we find that the interaction between two wrapped cylinders may either be attractive or repulsive, depending on their relative orientation along the elastic filament. The details of the interaction are however different from those found on the simple Gaussian level.

In another recent related work, Koslover and Spakowitz ${ }^{35}$ studied the role of local DNA twist in the coupling between bound proteins. It was shown that twist resistance results in a more complex interaction between the bound proteins exhibiting, e.g., damped oscillations superimposed over and counteracting the attractive protein-protein interaction. In a different context, Sudhanshu et al. ${ }^{36}$ considered the spatial orientation of the nucleosome spool with wrapped DNA chains and showed that this orientational degree of freedom for the spool modifies the tension-extension curve in a pulling experiment due to the unwrapping transitions of the DNA chain from the nucleosome. In this paper, confining ourselves to the 2D Eulerian plane, we therefore neglect the contribution of the polymer twist as well as bound spool spatial orientation and focus primarily on exact solutions for the case where the polymer is described as a twist-free worm-like chain and the axis of the wrapped spools is forced to stay perpendicular to the Eulerian plane.

The organization of the paper is as follows: In Sec. II, we define our model and discuss the general formalism which we shall employ in our study. In Sec. III, we apply our formalism to the problem of the unwrapping transition for a single wrapped cylinder and in Sec. IV, we study the unwrapping transition of the filament from two cylinders. In Sec. V, we consider the problem of effective interaction mediated by the elastic filament's fluctuations between two wrapped cylinders. We summarize our results and conclude in Sec. VI.

\section{FILAMENT WRAPPING AROUND CYLINDERS: MODEL AND FORMALISM}

\section{A. Filament elastic energy}

Consider an inextensible semi-flexible filament of total arc-length $L$ confined to a 2D Eulerian plane with coordinates $(x, y)$. The tangent vector of the filament is normalized to one and can thus be parametrized as $\mathbf{t}(s)=\dot{\mathbf{x}}(s)$ $=(\cos (\psi(s)), \sin (\psi(s))$. In this parametrization the inextensibility constraint is taken into account exactly. The bending energy of the filament is given by

$$
\int_{0}^{L} d s \frac{\kappa}{2}\left(\frac{d^{2} \mathbf{x}}{d s^{2}}\right)^{2}=\int_{0}^{L} d s \frac{\kappa}{2}\left(\frac{d \psi}{d s}\right)^{2},
$$

where $\kappa$ is the filament stiffness parameter and has dimensions of energy times length (the so-called "persistence length" is defined as $L_{p}=\kappa /\left(k_{B} T\right)$ for the ambient temperature $T)$. We assume that one end of the filament is fixed at $\mathbf{x}(s=0)=0$ and the other end is pulled with an external 


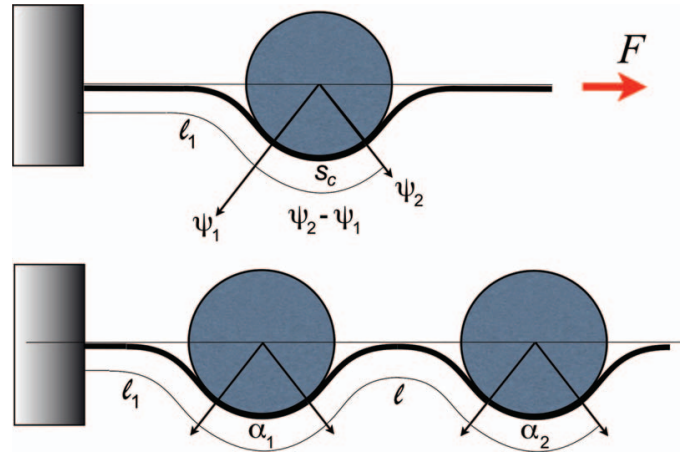

FIG. 1. A schematic drawing of the geometry as well as various quantities defined in the text for one and two cylinders wrapped on the Eulerian filament (shown in a symmetric mode). Specifically, for one cylinder (top) the angle at which the filament first touches the cylinder (advancing along the arc-length to the cylinder) is $\psi_{1}$ and the angle at which it leaves is $\psi_{2}$. For two cylinders (bottom), separated by a distance $l$, the contact angles $\alpha_{1}$ and $\alpha_{2}$ are as shown in the figure.

tension $\mathbf{F}$ in the direction $x$, see Fig. 1. The potential energy of the filament is thus given by

$$
-F x(L)=-F \int_{0}^{L} d s \cos (\psi(s)) .
$$

The total energy of the filament without any interactions with objects in the plane is then given as an elastic energy functional $E[\psi]$ as

$$
E(L)=E[\psi]=\int_{0}^{L} d s\left[\frac{\kappa}{2}\left(\frac{d \psi}{d s}\right)^{2}-F \cos (\psi(s))\right],
$$

which is the standard Euler elastic energy expression for the elastic filament under external force.

The energy function Eq. (3) also arises in the study of parallel cylinders adhering to elastic membranes. ${ }^{37-40}$ In this case, when the cylinders are parallel, and if only membrane height fluctuations in the direction normal to the cylinders are taken into account, the problem becomes effectively onedimensional and if one uses the Helfrich Hamiltonian for the energy of the membrane due to its height fluctuations one can write (up to a constant term) $)^{39}$

$$
E_{\text {memb }}=\mathcal{L} \int_{0}^{L} d s\left[\frac{\kappa}{2}\left(\frac{d \psi}{d s}\right)^{2}-\sigma \cos (\psi(s))\right],
$$

where $\psi(s)$ is the tangent angle of the membrane in the direction perpendicular to the cylinders ( $s$ being the coordinate in this direction), $L$ is the length of membrane in this direction, and $\mathcal{L}$ the length parallel to the cylinders. The term $\kappa$ is the membrane bending rigidity and $\sigma$ the surface tension. The Hamiltonian Eq. (4) is thus formally the same as that studied by Rudnick and Bruinsma ${ }^{5}$ as given by Eq. (3); however there are a number of notable and crucial differences between the two models. First, Eq. (4) is multiplied by a macroscopic quantity $\mathcal{L}$, this means that the statistical mechanics can be determined by purely energetic (or mean field) considerations by minimizing Eq. (4). In this limit one can also assume that fluctuations are small and thus fluctuations can be taken into account via an expansion about the minimal energy configuration and treated harmonically as was done by
Rudnick and Bruinsma ${ }^{5}$ for the polymer model. Another technical difference is that when one considers the interaction between the cylinders in the membrane model, the force is calculated as a function of the spatial distance between the cylinders, whereas in the polymer problem it is more natural to consider the arc-length along the polymer separating the cylinders as the relevant physical parameter. However within the formalism introduced here both ensembles (fixed spatial distance and fixed arc-length) can be handled on an equal footing. This choice of ensemble is especially important when analyzing the effect of fluctuations. The above points thus conclude the technical motivation for our study: as the problem is effectively one-dimensional, one can be in a limit where the effects of fluctuations are not merely perturbative and a full treatment of fluctuations is thus necessary. The limit where the energetic minimization is valid in the treatment of Eq. (3) is when $\kappa$ and $F$ are large (as compared with $k_{B} T$ ) while their ratio is kept fixed.

We also emphasize that our model is one where the size of the objects that are wrapped, the cylinders, is large with respect to the microscopic details of the polymer. The effect of absorbed objects on a more discrete model has been studied in Ref. 41, where in particular the effect of absorbed objects on the effective persistence length was taken into account.

\section{B. Wrapping adhesive energy}

Consider now a cylinder embedded in the plane which is free to move and which can become attached to the elastic filament. It is important that the cylinder can move since the position at which the filament is attached to the cylinder is not constrained in space. We assume that there is a favorable energy of interaction between the cylinder and the filament which is local and proportional to the arc-length of the filament touching the cylinder. We further assume that in the interaction region between the filament and the cylinder the former follows the surface of the cylinder exactly and that the energy of interaction is consequently given by

$$
E_{i}=-\gamma s_{c},
$$

where $\gamma$ is a line tension parametrizing the affinity of the filament for the cylinder and $s_{c}$ is the arc-length of the filament which is attached to the cylinder. If the angle at which the filament first touches the cylinder (advancing along the arclength to the cylinder) is $\psi_{1}$ and the angle at which it leaves is $\psi_{2}$ (see Fig. 1, top), then the length of the filament touching the cylinder is $s_{c}=R\left|\psi_{2}-\psi_{1}\right|$ and thus

$$
E_{i}=-\gamma R\left|\psi_{2}-\psi_{1}\right|
$$

Note that if $\psi_{2}>\psi_{1}$ the filament wraps anti-clockwise and if $\psi_{2}<\psi_{i}$ then it wraps clockwise around the filament. As well as having a surface interaction term, there is also a mechanical bending (potential) energy associated with the arc-length following the contour of the cylinder. This mechanical energy is given by $E_{m}=E\left(s_{c}\right)$, where the energy functional is defined in Eq. (3). The path that follows the cylinder arriving at angle $\psi_{1}$ and leaving at angle $\psi_{2}$ is parametrized over $\left[0, s_{c}\right]$ as $\psi_{s}=\left(\psi_{2}-\psi_{1}\right) s / s_{c}+\psi_{1}$. Substituting this trajectory into 
Eq. (3) yields

$$
E_{m}=\frac{\kappa}{2 R}\left|\psi_{2}-\psi_{1}\right|-F R \operatorname{sgn}\left(\psi_{2}-\psi_{1}\right)\left[\sin \left(\psi_{2}\right)-\sin \left(\psi_{1}\right)\right] .
$$

The total energy of the system is then composed of a bulk elastic term, Eq. (3), for the free segments of the filament and a boundary or contact interaction energy $E_{c}$ with the cylinder which is a sum of the terms in Eqs. (6) and (7), i.e., $E_{c}=E_{i}$ $+E_{m}$.

One notes that the above energy functional is analogous to a 1D Coulomb fluid with a charged boundary, but with an imaginary surface charge. ${ }^{42,43}$

\section{Partition function of a filament-cylinder complex}

To determine the conformational equilibrium of a system consisting of an elastic filament with any number of wrapped cylinders we must evaluate the corresponding partition function. To do this we first define the evolution kernel $K\left(\psi, \psi^{\prime}, s\right)$ which evolves the state of the filament for a distance $s$ measured along its arc-length with the boundary condition that $\psi(0)=\psi$ and $\psi(s)=\psi^{\prime}$. This kernel is defined by

$$
K\left(\psi, \psi^{\prime}, s\right)=\int_{\psi(0)=\psi}^{\psi(s)=\psi^{\prime}} d[\psi] \exp (-\beta E[\psi]),
$$

where $\beta=1 / k_{\mathrm{B}} T$ and $E[\psi]$ is the elastic energy functional in Eq. (3). It determines the evolution in arc-length $s$ of a wave function $f(\psi)$ by

$$
\int d \psi^{\prime} K\left(\psi, \psi^{\prime}, s\right) f\left(\psi^{\prime}\right)=\exp (-s H) f(\psi),
$$

where $H$ is the corresponding Hamiltonian operator

$$
H=-\frac{1}{2 \beta \kappa} \frac{d^{2}}{d \psi^{2}}-\beta F \cos (\psi) .
$$

The partition function for a single cylinder, which first becomes attached to the filament at arc-length $s=l_{1}$ measured from the fixed end $\mathbf{x}(s=0)=0$ (see Fig. 1), is then given by

$$
\begin{aligned}
Z= & \int d \psi_{0} d \psi_{1} d \psi_{2} d \psi_{3} K\left(\psi_{0}, \psi_{1}, l_{1}\right) S\left[\psi_{1}, \psi_{2}\right] \\
& \times K\left(\psi_{2}, \psi_{3}, L-l_{1}-R\left|\psi_{2}-\psi_{1}\right|\right)
\end{aligned}
$$

where $\psi(0)=\psi_{0}$ and $\psi(L)=\psi_{3}$ and the definition of $S$ follows from the contact energy (or the interaction energy between the cylinder and the filament as defined in Sec. II B) and is given by

$$
\begin{aligned}
S\left[\psi_{1}, \psi_{2}\right]= & \exp \left(\beta\left|\psi_{2}-\psi_{1}\right|\left(\gamma R-\frac{\kappa}{2 R}\right)\right. \\
& \left.+\beta F R \operatorname{sgn}\left(\psi_{2}-\psi_{1}\right)\left[\sin \left(\psi_{2}\right)-\sin \left(\psi_{1}\right)\right]\right) .
\end{aligned}
$$

The generalization to a system in which the elastic filament interacts with several wrapped cylinders is obvious from the above.

In the limit of a long elastic filament where the cylinders are close to its midpoint, we make use of the fact that the propagator at large arc-lengths is given in terms of the ground-state wave function of the corresponding Schrödinger equation

$$
K\left(\psi, \psi^{\prime}, L\right)=\exp \left(-E_{0} L\right) \Psi_{0}(\psi) \Psi_{0}\left(\psi^{\prime}\right),
$$

where $E_{0}$ is the ground-state energy of the Hamiltonian $H$, Eq. (10), and $\Psi_{0}$ the corresponding wave function, i.e., $H \Psi_{0}(\psi)=E_{0} \Psi_{0}(\psi)$. The ground-state energy in the groundstate-dominance limit then gives also the partition function in the limit of large arc-lengths.

\section{Constrained and free wrapping of cylinders}

Because of specific interactions between the filament and the cylinders the wrapping angles $\psi_{1}$ and $\psi_{2}$ may not be completely free and constraints may exist that reduce their overall degrees of freedom. For instance, in the limit where the adhesion energy $\gamma$ is very large, the elastic filament could wrap around the cylinder a number of times, via an escape into the third dimension that does not cost any extra energy, or so we assume in this study.

The simplest constraint is to take the wrapping angle (see Fig. 1, top) $\psi_{2}-\psi_{1}=\alpha_{1}$ fixed. The constrained partition function at a fixed wrapping angle can be computed and then the ensemble where it varies can be obtained by carrying out the remaining integrations with the appropriate statistical weights. This holds for a single wrapped cylinder but can be extended to the case of several cylinders as well.

For a single cylinder we then define the constrained partition function for the ensemble where $\psi_{2}-\psi_{1}=\alpha_{1}$ is kept fixed as

$$
\begin{aligned}
Z\left(\alpha_{1}\right)= & \int d \psi_{0} d \psi_{1} d \psi_{3} K\left(\psi_{0}, \psi_{1}, l_{1}\right) \\
& \times \exp \left(\beta F R \operatorname{sgn}\left(\alpha_{1}\right)\left[\sin \left(\psi_{1}+\alpha_{1}\right)-\sin \left(\psi_{1}\right)\right]\right) \\
& \times K\left(\psi_{1}+\alpha_{1}, \psi_{3}, L-l_{1}-R\left|\alpha_{1}\right|\right) .
\end{aligned}
$$

The partition function for the ensemble where the contact angle can vary freely is consequently given by

$$
Z=\int_{-L-l_{1} / R}^{L-l_{1} / R} d \alpha_{1} Z\left(\alpha_{1}\right) \exp \left(\beta\left|\alpha_{1}\right|\left(\gamma R-\frac{\kappa}{2 R}\right)\right) .
$$

Note that given that the point of first contact between the cylinder and filament is at $s=l_{1}$, the maximum amount of filament that can be wrapped about the cylinder is $L-l_{1}$, corresponding to wrapping angles of $\pm\left(L-l_{1}\right) / R$ (clockwise and anti-clockwise). When $\alpha_{1}$ is fixed, the surface terms are periodic in $\psi_{1}$ and one can use standard Mathieu function analysis to carry out the computations. The procedure is described in detail in Appendix A.

For two cylinders separated by a distance $l$, where the first one is located an arc-length $l_{1}$ away from the fixed end and with contact angles $\alpha_{1}$ and $\alpha_{2}$ (see Fig. 1, bottom), we have in complete analogy with above

$$
\begin{aligned}
Z\left(\alpha_{1}, \alpha_{2}\right)= & \int d \psi_{0} d \psi_{1} d \psi_{3} d \psi_{5} K\left(\psi_{0}, \psi_{1}, l_{1}\right) \mathcal{S}_{R_{1}}\left(\psi_{1}, \alpha_{1}\right) \\
& \times K\left(\psi_{1}+\alpha_{1}, \psi_{3}, l\right) \\
& \times \mathcal{S}_{R_{2}}\left(\psi_{3}, \alpha_{2}\right) K\left(\psi_{3}+\alpha_{2}, \psi_{5}, L_{12}\right)
\end{aligned}
$$

where

$$
L_{12}=L-l-l_{1}-R_{1}\left|\alpha_{1}\right|-R_{2}\left|\alpha_{2}\right|
$$


is the arc-length between the right, free, end of the chain, and the rightmost cylinder (see Fig. 1, bottom), and

$$
\mathcal{S}_{R}(\psi, \alpha)=\exp (\beta F R \operatorname{sgn}(\alpha)[\sin (\psi+\alpha)-\sin (\psi)]),
$$

where $R_{1}$ is the radius of cylinder 1 and $R_{2}$ is that of cylinder 2 .

\section{E. Constrained arc-length separation between wrapped cylinders}

Now consider the limit of a long filament where the cylinders are close to the midpoint, i.e., we take $l_{1}$ and $L-l_{1}$ large while keeping the arc-length between the final point of the filament touching cylinder 1 and the first touching cylinder $2, l$, constant (see Fig. 1, bottom). These are all lengths along the chain (arc-length) which are fixed, if we wish to keep the physical distance fixed in a given direction we must consider another ensemble; we shall discuss this later. In this limit (corresponding to the limit of ground state dominance, Eq. (13)), we find, up to an overall factor,

$$
\begin{aligned}
Z\left(\alpha_{1}, \alpha_{2}\right)= & \exp \left(-E_{0} L_{12}\right) \int d \psi_{1} d \psi_{3} \Psi_{0}\left(\psi_{1}\right) \mathcal{S}_{R_{1}}\left(\psi_{1}, \alpha_{1}\right) \\
& \times K\left(\psi_{1}+\alpha_{1}, \psi_{3}, l\right) \mathcal{S}_{R_{2}}\left(\psi_{3}, \alpha_{2}\right) \Psi_{0}\left(\psi_{3}+\alpha_{2}\right) .
\end{aligned}
$$

An interesting case emerges when the two wrapping angles are equal and correspond to complete single wrapping, $\alpha_{1}=\alpha_{2}=2 \pi$. The $l$ dependent part of the partition function is then given by

$$
\begin{aligned}
Z(l)= & \exp \left(E_{0} l\right) \int d \psi_{1} d \psi_{3} \Psi_{0}\left(\psi_{1}\right) \\
& \times K\left(\psi_{1}+2 \pi, \psi_{3}, l\right) \Psi_{0}\left(\psi_{3}+2 \pi\right)
\end{aligned}
$$

and using the periodicity this gives

$$
Z(l)=\exp \left(E_{0} l\right) \int d \psi_{1} d \psi_{3} \Psi_{0}\left(\psi_{1}\right) K\left(\psi_{1}, \psi_{3}, l\right) \Psi_{0}\left(\psi_{3}\right) .
$$

The same result is found if one assumes that the wrapping is antisymmetrical, $\alpha_{2}=-\alpha_{1}=-2 \pi$. The symmetry of wrapping between the two cylinders will be addressed further below. The difference between symmetric and antisymmetric wrapping is obvious from Figs. 1 and 2.

\section{F. Wrapping-unwrapping transition of cylinders on an elastic filament}

We first consider the case of a single adsorbing cylinder. The elastic filament is of length $L$ and a wrapped cylinder is positioned at $l_{1}$. We restrict ourselves to the case where both $L$ and $l_{1} \rightarrow \infty$, assuming that $l_{1}$ is not fixed so that the cylinder can attach anywhere and consider the case where the elastic filament can wrap on the cylinder any number of times.

In this case, the expression in Eq. (14) becomes, up to a constant prefactor, independent of $\alpha$ :

$$
\begin{aligned}
& Z(\alpha)=\exp \left(-E_{0}(L-R|\alpha|)\right) f(\alpha), \quad \text { with } \\
& f(\alpha)=\int_{0}^{2 \pi} d \psi \Psi_{0}^{2}(\psi) \mathcal{S}_{R}(\psi, \alpha),
\end{aligned}
$$
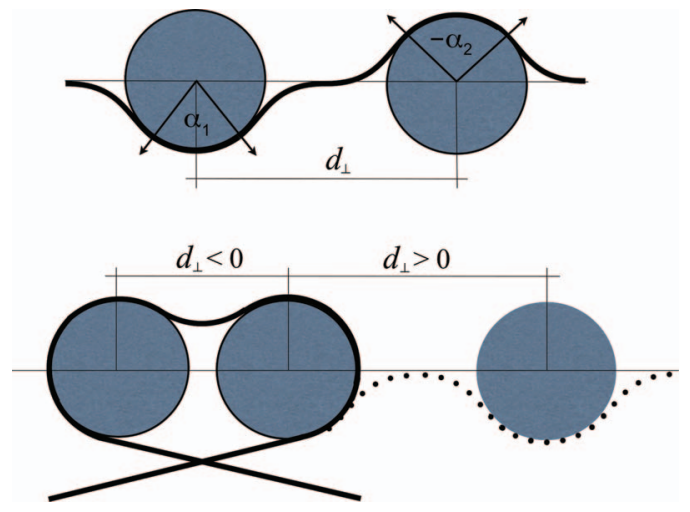

FIG. 2. Top: Schematic view of the wrapping of two cylinders in an antisymmetric mode where the wrapping angles for the two cylinders differ in sign. Bottom: The symmetric looped configuration (full line) of two wrapped cylinders corresponds to a negative value of the horizontal projected separation $d_{\perp}$ (i.e., cylinder 2 lies to the left of cylinder 1) and the symmetric extended configuration (dotted line) with positive $d_{\perp}$ (i.e., cylinder 2 lies to the right of cylinder 1).

and the function $f(\alpha)$ is clearly bounded. From Eq. (15) the partition function for the ensemble with variable $\alpha$ is obtained by integrating Eq. (22) over $\alpha$ from $-L / R$ to $+L / R$, i.e.,

$$
\begin{aligned}
Z= & \exp \left(-L E_{0}\right) \int_{-L / R}^{L / R} d \alpha \\
& \times \exp \left(\beta|\alpha|\left(\gamma R-\frac{\kappa}{2 R}+\frac{R E_{0}}{\beta}\right)\right) f(\alpha) .
\end{aligned}
$$

Defining

$$
\Delta E=\gamma R-\frac{\kappa}{2 R}+\frac{R E_{0}}{\beta},
$$

we see that the elastic filament will then wrap around the cylinder a macroscopic number of times (in the sense that the total length wrapped around the filament will be of the order of the filament length) if $\Delta E>0$. However, for $\Delta E<0$ the filament will have only a microscopic length wrapped around the cylinder. The equation $\Delta E=0$ therefore defines a wrapping transition in the phase diagram of variables $\beta$ and $F$ as a consequence of competition between the wrapping energy of the cylinder and the configurational entropy of the chain.

In the zero-temperature limit where $\beta \rightarrow \infty$ the ground state of the filament configuration is a straight line in the direction of the applied force and so without a cylinder

$$
Z(L) \approx \exp \left(-L E_{0}\right)=\exp (\beta F L),
$$

or $E_{0}(T=0)=-\beta F$. Therefore, if $\gamma R-\kappa / 2 R-F R>0$, the system is in the wrapped state, otherwise the filament unwraps from the cylinder. This conclusion is easy to deduce from purely energetic arguments for large values of the wrapping angle $\alpha$.

We next consider several cylinders in the plane and assume that the filament can wrap around all of them without impediment. The wrapping will induce effective interactions between the cylinders that can either be repulsive, attractive, or non-monotonic. Wrapping transitions with effective interactions between cylinders can be viewed as a model for 
nucleosomal wrapping. ${ }^{12,13,21}$ However, the details of the most general case remain to be elaborated.

For clarity we briefly describe the system with three cylinders: cylinder 1 at position $l_{01}$ from the left end of the chain, cylinder 2 separated from 1 by a distance $l_{12}$, cylinder 3 separated from 2 by a distance $l_{23}$ and with contact angles $\alpha_{1}, \alpha_{2}$, and $\alpha_{3}$. The partition function can be written as

$$
\begin{aligned}
Z\left(\alpha_{1}, \alpha_{2}, \alpha_{3}\right)= & \int d \psi_{0} d \psi_{1} d \psi_{3} d \psi_{5} d \psi_{7} K\left(\psi_{0}, \psi_{1}, l_{01}\right) \mathcal{S}\left(\psi_{1}, \alpha_{1}\right) K\left(\psi_{1}+\alpha_{1}, \psi_{3}, l_{12}\right) \mathcal{S}\left(\psi_{3}, \alpha_{2}\right) \\
& \times K\left(\psi_{3}+\alpha_{2}, \psi_{5}, l_{23}\right) \mathcal{S}\left(\psi_{5}, \alpha_{3}\right) K\left(\psi_{5}+\alpha_{3}, \psi_{7}, L-\left(l_{01}+l_{12}+l_{23}+R_{1}\left|\alpha_{1}\right|+R_{2}\left|\alpha_{2}\right|+R_{3}\left|\alpha_{3}\right|\right)\right),
\end{aligned}
$$

with the general definition

$$
\mathcal{S}\left(\psi_{2 i+1}, \alpha_{i}\right)=\exp \left(\beta F R_{i} \operatorname{sgn}\left(\alpha_{i}\right)\left[\sin \left(\psi_{2 i+1}+\alpha_{i}\right)-\sin \left(\psi_{2 i+1}\right)\right]\right)
$$

where $i$ (for $i=0,1,2$ ) is the index of the wrapped cylinder.

The general system for $N$ particles bears some overall similarity to the 1D Tonks gas, although the effective interactions between the cylinders are more complicated. In order to make this system applicable to the problem of nucleosome wrapping around DNA, it may be necessary to insert a chemical potential term for the cylinders similar to the case of a grand-canonical Tonks gas. ${ }^{12,13}$ The main difference between the two models is, however, in the fact that the interaction between the wrapped cylinders along the elastic filament can be much more complicated than in the Tonks case and could in principle lead to "phase separation" without imposing any excluding energy barriers along the chain. ${ }^{21}$

\section{UNWRAPPING TRANSITION: ONE CYLINDER}

We now apply the general theory derived in Secs. II AII $\mathrm{F}$ to the problem of the unwrapping transition for a single wrapped cylinder.

To accommodate the wide range of values that the variables take in the physical systems to which our theory would be applicable, it is convenient to recast the calculation in terms of equivalent dimensionless variables. We consider an elastic filament of persistence length $L_{p}$, fixed at one end and pulled from the other end with a force $F$. The wrapped cylinders are all of radius $R$. We define the following dimensionless variables

$$
\mu=\frac{2 L_{p}}{R}, f=\beta R F, \sigma=\beta \gamma R, \varepsilon=E R,
$$

where $L_{p}=\beta \kappa$ and $\gamma$, the wrapping energy of a cylinder per unit of arc-length, and other parameters have been defined before, Eqs. (1)-(5). In terms of these variables the Hamiltonian can be written as $H=H^{\prime} /(\mu R)$ and thus $H^{\prime}$ has eigenvalues which can be written in the form of the Schrödinger equation

$$
H^{\prime} \psi_{m}=\mu \epsilon_{m} \psi_{m}
$$

where $\epsilon_{m}=E_{m} R$.

As an illuminating example let us consider a specific case with the dimensionless quantities $\mu=1$ and $\sigma=0.5$. Assuming cylinder radius of $R=2 \mathrm{~nm}$, this gives actual parameter values as $L_{p}=1 \mathrm{~nm}$ (persistence length), $\kappa=k_{B} T \times L_{p} \simeq 4$ $\times 10^{-30} \mathrm{~J} \mathrm{~m}$ (bending stiffness) at $T=300 \mathrm{~K}$, and $\gamma=1 \mathrm{pN}$ (line tension for cylinder-filament adhesion).

For the single cylinder wrapping transition, a rough estimate for its occurrence can be obtained in the following way. In Sec. II F, it was argued that for large $\beta$ (low temperature) $E_{0} \sim \beta F$ and that the wrapped phases occurs for forces which obey $\Delta E>0$, Eq. (24). For scaled quantities as introduced above this inequality becomes

$$
f<\sigma-\frac{\mu}{4} \quad \text { and thus } \quad \sigma^{\prime} \equiv \sigma-\frac{\mu}{4}>0 .
$$

Alternatively, we can then consider the Schrödinger equation, Eq. (29) (see also Eq. (10)), as an approximate oscillator equation and get an improved estimate $E_{0}=-\beta F+\sqrt{F /(4 \kappa)}$. Using Eq. (30) above, the condition for the wrapping transition then becomes

$$
f-\sqrt{f / 2 \mu}-\sigma^{\prime}<0 .
$$

This can be recast as a quadratic equation in $\sqrt{f}$ and the condition can be satisfied if the discriminant is positive, i.e.,

$$
\sigma^{\prime}>-\frac{1}{8 \mu} \text { and thus } \sigma>\frac{\mu}{4}-\frac{1}{8 \mu} .
$$

In the limit where $\mu$ is large, the above expression reduces to the condition in Eq. (30). There will of course be corrections to this form due to the non-harmonic terms in the effective potential in Eq. (29). Nevertheless, from both arguments it follows that a sufficient condition for the transition to occur is $\sigma>\mu / 4$. If we return to original variables we see that the second condition of Eq. (32) gives

$$
\gamma>\gamma^{\prime}, \quad \text { where } \quad \gamma^{\prime}=\frac{1}{2} \frac{\kappa}{R^{2}}-\frac{\left(k_{B} T\right)^{2}}{16 \kappa} .
$$

In the limit of zero temperature this result agrees with that of Weikl $^{37}$ for a cylinder and membrane. The interpretation of the zero-temperature result is simply that the adhesive energy must overcome the bending energy to make the bound state stable. We see that the effect of temperature is to diminish the minimum adhesion energy necessary to obtain a bound 


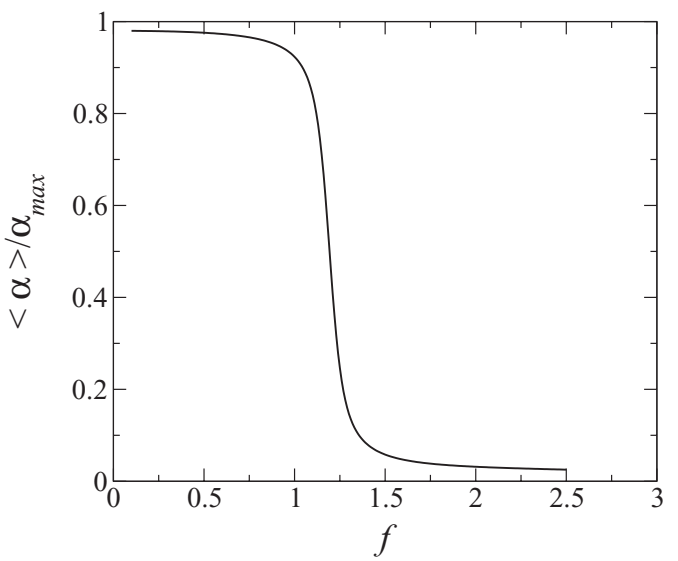

FIG. 3. The wrapping transition of a single cylinder on an elastic filament. Exact solution for the average wrapping angle ratio $\langle\alpha\rangle / \alpha_{\max }$ is shown as a function of the dimensionless external tension $f=\beta R F$ for fixed $\mu=1$ and $\sigma=0.75$.

phase. At first sight this appears counter-intuitive and so we investigate the effect of temperature below in more detail.

We first present an exact calculation of the wrapping transition in a particular case. The mean value of $\alpha$, i.e., $\langle\alpha\rangle$, is calculated for fixed $(\mu, \sigma)$ as a function of the reduced force $f$. Whilst taking the filament to be of infinite extent we restrict the range of $\alpha$ to be $-\alpha_{\max }<\alpha<\alpha_{\max }$ and take $\alpha_{\max }=$ 100. For small $f$ we expect $\langle\alpha\rangle$ to be large, which corresponds to the elastic filament maximally wrapped onto the cylinder: $|\alpha|=\alpha_{\max }$. As $f$ is increased the system will go through a transition from the wrapped to the unwrapped configuration.

To solve the corresponding Schrödinger equation, the range of $x$ is chosen to be an integer multiple $N$ of $2 \pi$, where $N$ would then be the number of lattice sites in the Bravais lattice for the discretized Hamiltonian. The Schrödinger equation (Eq. (29)) is then recast as a matrix equation with imposed periodic boundary conditions. This will lead to a band structure with the Brillouin zone and the number of Bloch states per band determined by $N$. Since the solution of this problem requires only strictly periodic eigenfunctions we choose $N=1$ and the range of $x$ as $2 \pi$. The eigenvalues are then equal to $\mu \epsilon_{m}$.

In Fig. 3 we show the results for the mean wrapping angle $\langle\alpha\rangle$, scaled by $\alpha_{\max }$, as a function of the external dimensionless force $f$ for fixed $\mu=1$ and $\sigma=0.75$. The wrapping transition, corresponding to the maximum in the derivative $\partial\langle\alpha\rangle / \partial f$, then occurs for the critical reduced force $f_{c} \simeq 1.2$ which, for the parameters stated above (i.e., using $L_{p}=1 \mathrm{~nm}, R=2 \mathrm{~nm}$ ) gives $F_{c} \simeq 2.4 \mathrm{pN}$. Compare this with the corresponding estimates from

(i) Eq. (30). We find

$$
f_{c}=\sigma-\mu / 4=0.5 \rightarrow F_{c}=1 \mathrm{pN} .
$$

(ii) Eq. (31). We solve the quadratic equation (for $\mu=1$ and $\sigma=0.75$ ) to get

$$
f_{c}=1.31 \rightarrow F_{c}=2.67 \mathrm{pN} .
$$

Obviously, in order to get an accurate prediction for the critical force it is necessary to solve the full Schrödinger equa- tion as we did above. Nevertheless, the harmonic approximation in Eq. (31) is in acceptable agreement with this exact result.

In order to highlight the effect of temperature it is important to consider the dependence of $\langle\alpha\rangle$ on the force $F$ rather than on the dimensionless reduced force $f$ because the latter contains a hidden dependence on $T$ which would obscure the effect we are studying. In the harmonic approximation, we have that the critical force $F_{c}$ is predicted to be

$$
\sqrt{F_{c}}=\frac{k_{B} T}{4 \sqrt{\kappa}}+\left(\gamma-\gamma^{\prime}\right)
$$

We investigate two cases and take typical values for the parameters similar to those stated at the beginning of this section, i.e., $R=2 \mathrm{~nm}, L_{p}=1 \mathrm{~nm}, \kappa=k_{B} T \times L_{p}$ and the temperature is taken as $T=300 \mathrm{~K}$, so that $\mu=1$. We study two cases corresponding to $\sigma / \mu=0.15$ and 0.35 , respectively. We also note that these choices, respectively, give $\sigma^{\prime}>0$ and $\sigma^{\prime}<0$, which allows us to test the significance of the inequality in Eq. (32). We study a wide range of temperatures above and below $T=300 \mathrm{~K}$ which is necessary to reveal the $T$-dependence of the wrapping transition. Of course, such a wide temperature range does not occur in vivo but our aim is to test the prediction inferred from Eq. (34), namely that the critical force for the wrapping transition increases, rather than decreases, with increasing $T$. The values for $T$ considered are between $T=210$ and $390 \mathrm{~K}$ in intervals of $30 \mathrm{~K}$, and the results are shown in Fig. 4 for the above two cases:

(i) $\sigma / \mu=0.15, \sigma^{\prime}<0$ : This corresponds to relatively weak adhesion coefficient $\gamma=0.30 \mathrm{pN}$ and whilst we expect a wrapping transition to occur, we expect it to be relatively smooth and, according to Eqs. (33) and (34), to turn on more strongly as $T$ increases and for the critical force to increase as $T$ increases. We see these features in Fig. 4, left panel. We conclude also that even though $\sigma^{\prime}<0$ the wrapping transition does occur. For $T=300 \mathrm{~K}$ the harmonic approximation in Eq. (34) predicts $F_{c}=0.52 \mathrm{pN}$. The transition is not sharp and so it is not feasible to read a specific value for $F_{c}$ from the graph but it is clear that this prediction is very much on the low side.

(ii) $\sigma / \mu=0.35, \sigma^{\prime}>0$ : The adhesion coefficient $\gamma=0.70 \mathrm{pN}$ is larger and, in this case, we expect the transition to be stronger and sharper than in case (i). This is seen in Fig. 4, right panel. As $T$ increases the transition moves to larger critical force as predicted. For $T=300 \mathrm{~K}$ the harmonic approximation in Eq. (34) predicts $F_{c}=1.37 \mathrm{pN}$. From the graph the value of $F_{c}$ follows as $F_{c} \sim 3 \mathrm{pN}$, twice as big as the harmonic prediction.

We have thus verified that the effect of increasing temperature $T$ is to increase the critical force at which the wrapping transition occurs and this feature is clearly seen in Fig. 4(b). In other words we can conclude that increasing the temperature causes the wrapping transition to occur at larger force and the corollary is that for fixed force increasing the temperature can actually cause the transition: a somewhat surprising result! 

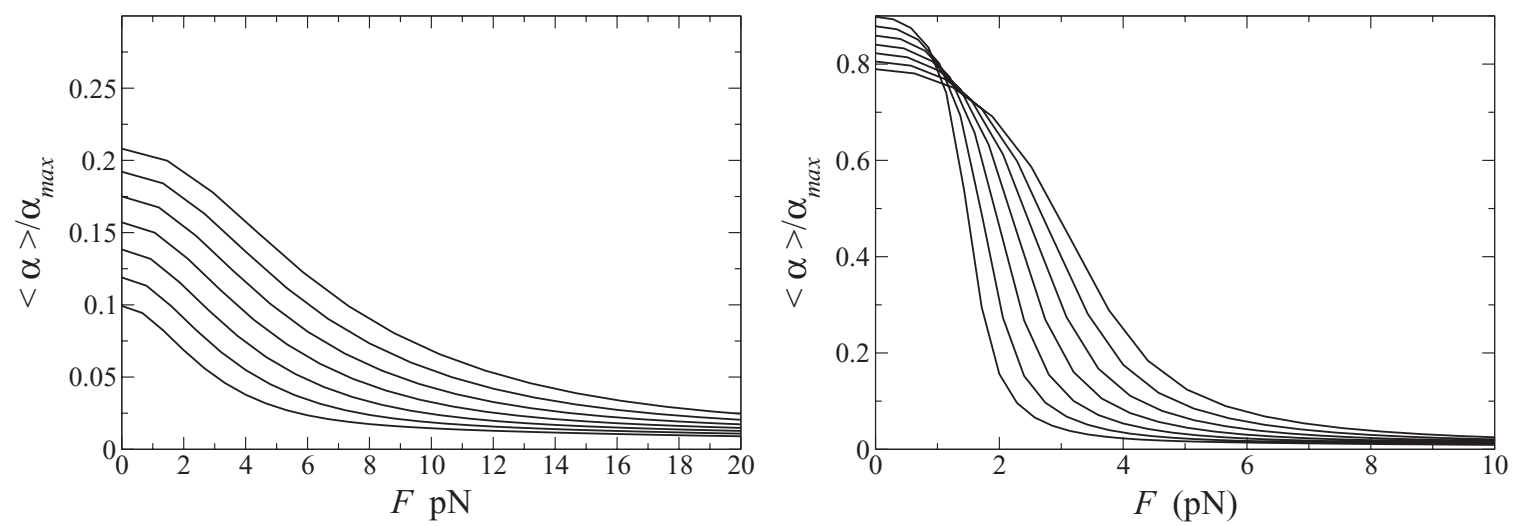

FIG. 4. The wrapping transition as a function of the actual force $F$ and for temperatures $T=210 \mathrm{~K}$ up to $390 \mathrm{~K}$ in steps of $30 \mathrm{~K}$. The physical values for the parameters are given in the text. In both cases the curves move right as $T$ increases. Left panel: we have $\sigma / \mu=0.15, \sigma^{\prime}<0$. The wrapping transition is relatively smooth and as $T$ increases it becomes stronger and moves to larger values of $F$. Right panel: $\sigma / \mu=0.35, \sigma^{\prime}>0$. The wrapping transition is much stronger and sharper as compared with the former case and, as predicted, the transition moves to larger values of $F$ as $T$ increases. It is this behavior in both cases which might be considered counter-intuitive.

These effects can be understood by noting that it is the ground-state energy $E_{0}$ for the full Schrödinger equation which contributes in Eq. (22) and this is clearly $T$-dependent and different from $E_{0}$ for the harmonic approximation. As $T$ increases the potential becomes narrower leading to $E_{0}$ increasing with $T$. This leads to a smaller threshold, $\gamma^{\prime}$, as defined in Eq. (33).

We finally note that the wrapping transition is controlled by the Mathieu ground state wavefunction which encodes the effect of fluctuations and is not Gaussian. However, from Eq. (10) we can estimate the relevance of the anharmonic terms in the potential and find that for $\beta F_{c} L_{p} \gg 1$ the harmonic oscillator approximation and hence the Gaussian approximation for the fluctuation contribution is good. This leads to the prediction for $F_{c}$ as given in Eq. (34). For our choice of parameters here, for which $\beta F_{c} L_{p} \sim 2$ it is evident from (ii) above that the harmonic approximation is not good. If we increase the value of $L_{p}$, say from $1 \mathrm{~nm}$ to $50 \mathrm{~nm}$ characteristic of dsDNA, and keeping other parameters fixed, then the relevant fluctuations are Gaussian. However, the quantity that matters is $F_{c} L_{p}$ and the key equation is Eq. (34) which can be rewritten as

$$
\sqrt{\beta F_{c} L_{p}}=\frac{1}{4}+\sqrt{\beta L_{p}}\left(\gamma-\gamma^{\prime}\right)
$$

So the Gaussian approximation is not good if the second term on the r.h.s. is $O(1)$ which is the case for

$$
\gamma \sim \frac{k_{B} T}{2}\left(\frac{L_{p}}{R^{2}}-\frac{1}{16 L_{p}}\right)
$$

Thus, the validity of the Gaussian approximation depends on the values $L_{p}, R$, and $\gamma$ and cannot be deduced from the value of any one of these parameters alone.

\section{UNWRAPPING TRANSITION: TWO CYLINDERS}

We now study the unwrapping transition in a system of two cylinders of radius $R$, labelled by $i=1,2$, wrapped by a length of filament. There are two cases which we consider.
The first case (case I) consists of the two cylinders wrapped by a fixed length of filament. The relevant variables, shown in Fig. 1, are as follows:

- The total angle, $\alpha_{i}$, wrapped by filament for the $i$ th cylinder. These are both fixed (quenched) for $i=1,2$.

- The length, $l$, of filament directly between the two cylinders, i.e., from the exit of the first cylinder to the entrance of the second, which is also fixed.

We encode the configuration of the cylinders by measuring the expectation value of $d_{\perp}$, the projected horizontal displacement between the centers of the two cylinders. The maximum projected horizontal displacement is given by $d_{\max }=l+2 R$. We shall deal with this case in Sec. IV A below.

The second case (case II) consists of the two cylinders pinned a distance $l^{\prime}$ apart along a filament, which are wrapped by a dynamical (or annealed) length of filament. The relevant variables are:

- The length $l^{\prime}$ of filament between the sites of pinning of the two cylinders. This is fixed (quenched).

- The total angle, $\alpha_{i}$, wrapped by filament for the $i$ th cylinder. These are dynamical (annealed) and free to change.

- The angle, $\alpha_{i}^{\prime}$, wrapped by the internal length of filament (initially of length $l^{\prime}$ ) between cylinders for the $i$ th cylinder. These are also dynamical; they take into account the ways in which the filament can wind.

- The length, $l$, of filament directly between the two cylinders, i.e., from the exit of the first cylinder to the entrance of the second. It is now dynamical being determined by $l=l^{\prime}-R\left(\alpha_{1}^{\prime}+\alpha_{2}^{\prime}\right)$.

Again we encode the configuration of the cylinders by measuring the expectation value of $d_{\perp}$, the projected horizontal displacement between the centers of the two cylinders. The maximum projected horizontal displacement is given now by $d_{\max }=l^{\prime}+2 R$. We shall deal with this case in Sec. IV B below. 


\section{A. Two cylinders: Constrained wrapping}

In the first case (case I) where the wrapping angles are fixed, we write the constrained partition function of this system as

$$
\begin{aligned}
Z\left(\alpha_{1}, \alpha_{2}, l\right)= & \int d \psi_{1} d \psi_{2}\langle 0| \hat{O}\left(\psi_{1}, \psi_{1}+\alpha_{1}\right) \\
& \times \exp (-H l / R \mu) \hat{O}\left(\psi_{2}, \psi_{2}+\alpha_{2}\right)|0\rangle,
\end{aligned}
$$

where we assume that the ground state dominates the external regions of the elastic filament, i.e., regions outside the part bounded by the two wrapped cylinders. The operator insertion $\hat{O}$ corresponds to the wrapping of the elastic filament around a cylinder and is given by

$$
\hat{O}(\psi, \psi+\alpha)=C_{\alpha}(\psi)|\psi\rangle\langle\psi+\alpha|,
$$

where

$$
C_{\alpha}(\psi)=\exp \left(|\alpha|\left[\sigma+\epsilon_{0}\right]+f \operatorname{sgn}(\alpha)[\sin (\psi+\alpha) \sin \psi]\right) .
$$

This gives for the constrained partition function Eq. (37)

$$
\begin{aligned}
Z\left(\alpha_{1}, \alpha_{2}, l\right)= & \int d \psi_{1} d \psi_{2} \Psi_{0}\left(\psi_{2}\right) C_{\alpha_{1}}\left(\psi_{1}\right) \sum_{m} e^{-\epsilon_{m} l / R} \\
& \times P_{m}\left(\psi_{1}+\alpha_{1}, \psi_{2}\right) C_{\alpha_{2}}\left(\psi_{2}\right) \Psi_{0}\left(\psi_{2}+\alpha_{2}\right),
\end{aligned}
$$

where

$$
P_{m}\left(\psi_{1}, \psi_{2}\right)=\Psi_{m}\left(\psi_{1}\right) \Psi_{m}\left(\psi_{2}\right) .
$$

The functions $P_{m}$ and the exponential in front of the $P_{m}$ can be pre-computed outside any nested integration loops for fixed $\alpha_{i}$, leading to a quick and straightforward evaluation of the above expression. We are interested in the average horizontal distance between the centers of the two cylinders which is given by

$$
\begin{aligned}
\left\langle d_{\perp}\right\rangle= & \frac{1}{Z\left(\alpha_{1}, \alpha_{2}, l\right)} \int d s \int d\left(\sin \psi_{s}\right) \int d \psi_{1} d \psi_{2}\left(\left\langle 0\left|\hat{O}\left(\psi_{1}, \psi_{1}+\alpha_{1}\right) e^{-H s / R \mu}\right| \psi_{s}\right\rangle\right. \\
& \left.\times\left\langle\psi_{s}\left|e^{-H(l-s) / R \mu} \hat{O}\left(\psi_{2}, \psi_{2}+\alpha_{2}\right)\right| 0\right\rangle+R \operatorname{sgn}\left(\alpha_{1}\right)\left\langle\sin \left(\psi_{2}\right)\right\rangle-R \operatorname{sgn}\left(\alpha_{2}\right)\left\langle\sin \left(\psi_{3}\right)\right\rangle\right) .
\end{aligned}
$$

A form suitable for numerical computations is reworked in Appendix B.

We remark at this point that in our model nothing stops the cylinders from passing through each other. Therefore the inter-cylinder force for small horizontal separations has to be interpreted only up to the limit of cylinders actually touching. The definition of this point depends on the symmetry of the wrapping, i.e., it differs when $\alpha_{1}=\alpha_{2}$ and when $\alpha_{1}=-\alpha_{2}$. These are the two cases that we have designated symmetric and antisymmetric, respectively.

The wrapping mediated interactions derived in this way should then be added to the hard core 1D Tonks gas model, ${ }^{44}$ which is what was only taken into account in the studies of the positional distribution of nucleosomes along the genome. ${ }^{12,13}$ We do not delve into this problem specifically here, but plan to address it elsewhere.

An interesting case with two cylinders was discussed in detail by Rudnick and Bruinsma: ${ }^{5}$ they dealt with a system composed of two cylinders at a fixed arc-length separation $l$ and with fixed wrapping angles, solved in the Gaussian approximation level. We analyze here the exact solution for the two phases described in Ref. 5 which are (see also Fig. 2):

(i) the looped phase where the mean projected separation $\left\langle d_{\perp}\right\rangle$ is negative meaning that the cylinder at larger distance along the filament lies to the left of the other cylinder, thus causing the elastic filament to loop and

(ii) the extended phase where $\left\langle d_{\perp}\right\rangle$ is positive and there is no loop.
We fix $\alpha_{1}$ and $\alpha_{2}$, and choose a range of elastic filament arclengths, $l$, between the cylinders. We calculate the average horizontal separation $\left\langle d_{\perp}\right\rangle$ for a given external tension $f$ (in dimensionless units) and plot $f$ versus $\left\langle d_{\perp}\right\rangle /(l+2 R)$ for various choices of parameters. The average horizontal separation is calculated from Eq. (42).

For symmetric wrapping described by $\alpha_{1}=\alpha_{2}=\pi$ and $\mu=10$ (high rigidity) the results are shown in Fig. 5, left panel. From left to right the lines are for $l=n R$ for integer $n=3 \ldots 8$. For small forces the filament is in the looped phase. Increasing the force actually makes the loops larger as shown by $\left\langle d_{\perp}\right\rangle$ becoming more negative. Eventually the extended phase becomes preferable and we have a limit of full extension as the force increases. Small $l$ also makes the loops more energetically favorable. For $\alpha_{1}=\alpha_{2}=\pi$ but smaller (rescaled) rigidity, $\mu=5$ (Fig. 5, right panel), we see that only the extended phase is allowed for the largest values of $l$. This implies that for a given rigidity there is a minimum distance (and obviously maximum force) for loop formation.

The largest extension in both these cases is noticeably less than the hypothetical maximum $d_{\max }=l+2 R$. This can be understood for the chosen fixed wrapping angle of $\pi$ since high rigidity constrains the filament to be close to tangential at both the entry and exit points.

In Fig. 6 we then plot $f$ versus $\left\langle d_{\perp}\right\rangle / d_{\max }$ where $d_{\max }=l$ $+2 R$ for $\mu=10$ (left) and $\mu=5$ (right) with fixed $\alpha_{1}=\alpha_{2}$ $=3 \pi / 8<\pi / 2$. The curves for the two rigidities are qualitatively similar with only the extended phase allowed. There is a small $l$ dependence, with curves corresponding to shorter $l$ 

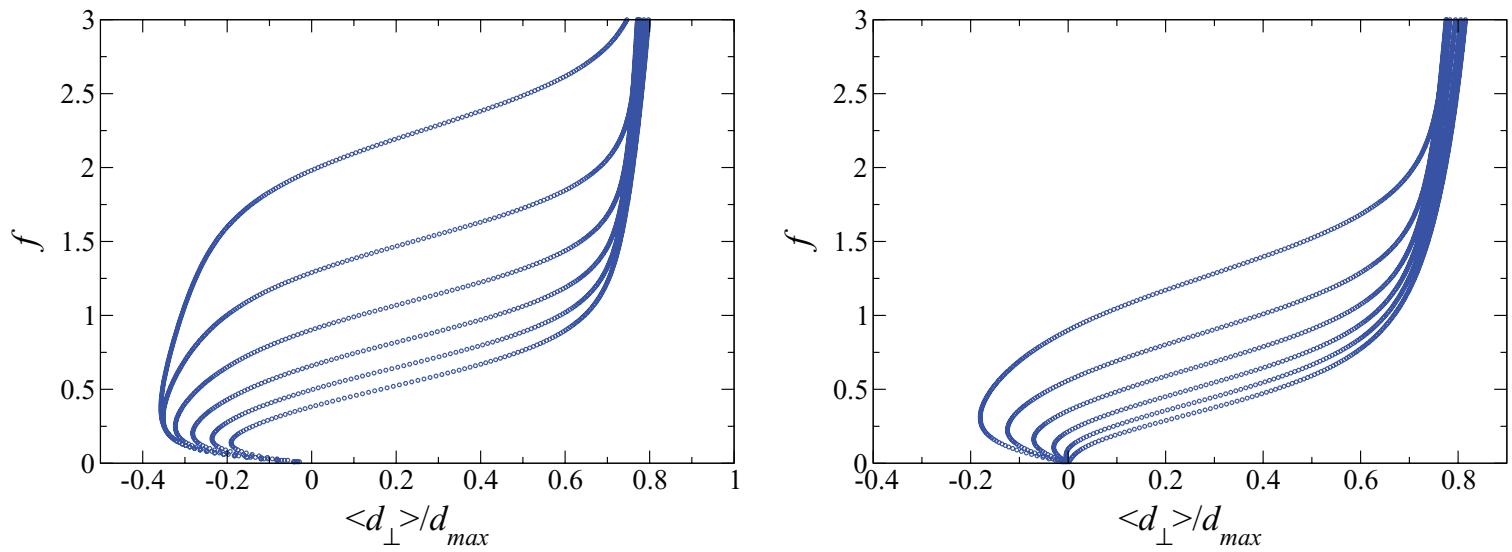

FIG. 5. Dependence of the average horizontal separation $\left\langle d_{\perp}\right\rangle$ (normalized to $d_{\max }=l+2 R$ ) on the external dimensionless tension $f=\beta R F$ for different sets of parameters in the case of constrained symmetric wrapping with $\alpha_{1}=\alpha_{2}=\pi$. Left panel: $\mu=10$ (high rigidity). Right panel: $\mu=5$ (low rigidity). Negative values of the average horizontal separation indicate the presence of a looped phase, i.e., the cylinder at larger distance along the elastic filament lies to the left of the other cylinder. Curves from left to right correspond to $l=n R$ for integer $n=3, \ldots, 8$.

lying to the right of those for longer $l$. Unlike in Fig. 5, the largest extension for both values of $\mu$ is now closer to $d_{\max }$ since the fixed wrapping angle is smaller than $\pi$ and the constraint that the filament is tangential to the cylinders has a weaker effect. We observe that as the rigidity decreases the dimensionless displacement for a given (small) external tension also decreases; a lower rigidity allows the elastic filament to fluctuate more and increase the entropic contribution to the free energy without incurring a large energy penalty. From our exact analysis, we thus qualitatively confirm the results for this system by Rudnick and Bruinsma. ${ }^{5}$

We will not discuss in detail the antisymmetric configuration $\alpha_{2}=-\alpha_{1}$ as here a looped phase does not emerge (though such configurations do occur with finite probability in the sum over the configurations but not on the average) and the plots are similar to those shown in Fig. 6.

Before we proceed with the case of unconstrained wrapping, it is important to consider the role of non-Gaussian fluctuations in the above analysis. For a given separation $l$ between the cylinders, the condition that qualitatively determines the relevance of the contribution of the $m$ th excited

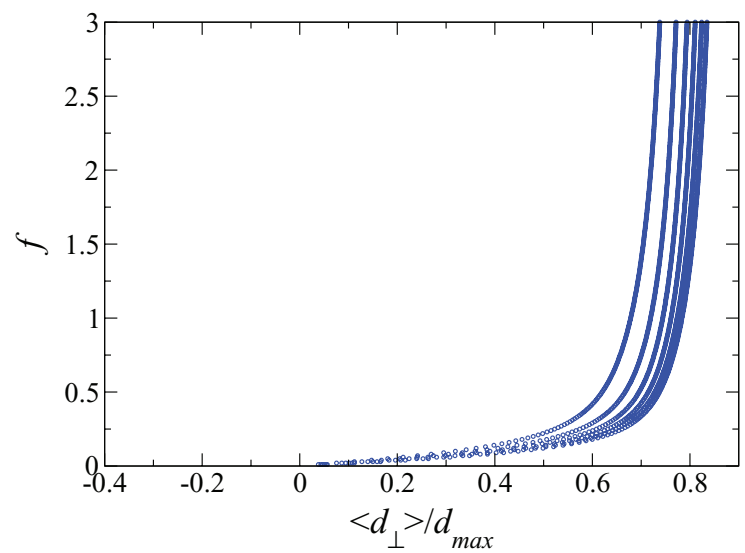

Mathieu state of energy $E_{m}$ to the evolution along the filament between the cylinders is $\left(E_{m}-E_{0}\right) l<1$. In the harmonic approximation this becomes $m \omega l<1$ where $\omega=\sqrt{\beta F /\left(4 L_{p}\right)}$ is the oscillator "frequency" in the harmonic approximation, which directly follows by expanding the nonlinear term in Eq. (10) to the second order. Using Eq. (10) and the variables defined in Eq. (28), we find that this translates to the condition

$$
2 m \frac{f}{\mu}\left(\frac{l}{R}\right)^{2}<1 \rightarrow 2 \frac{m f n^{2}}{\mu}<1
$$

where we have used $l=n R$ as shown in Fig. 5 where $n=3 \ldots 8$. In the left panel of Fig. 5, for which $\mu=10$, we deduce that for small $f$ the excited Mathieu states contribute significantly for $f<5 / n^{2}$; this corresponds to the onset of the looped phase and the behavior cannot be approximated only by the ground state contribution.

Another question is whether the harmonic approximation is nevertheless good when the excited states are included. From Sec. III, we note that the condition for the harmonic

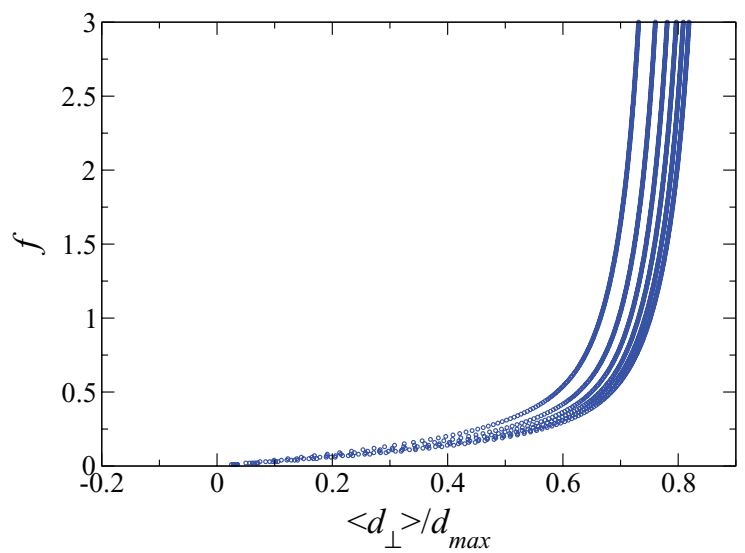

FIG. 6. Dependence of the average horizontal separation $\left\langle d_{\perp}\right\rangle$ (normalized to $d_{\max }=l+2 R$ ) on the external dimensionless force $f=\beta R F$ for different sets of parameters in the case of constrained symmetric wrapping $\alpha_{1}=\alpha_{2}=3 \pi / 8$. Left panel: $\mu=10$ (high rigidity). Right panel: $\mu=5$ (low rigidity). Clearly, for this set of parameters only the extended phase is allowed for large values of force regardless of rigidity. Curves from left to right correspond to $l=n R$ for integer $n=3, \ldots, 8$. 

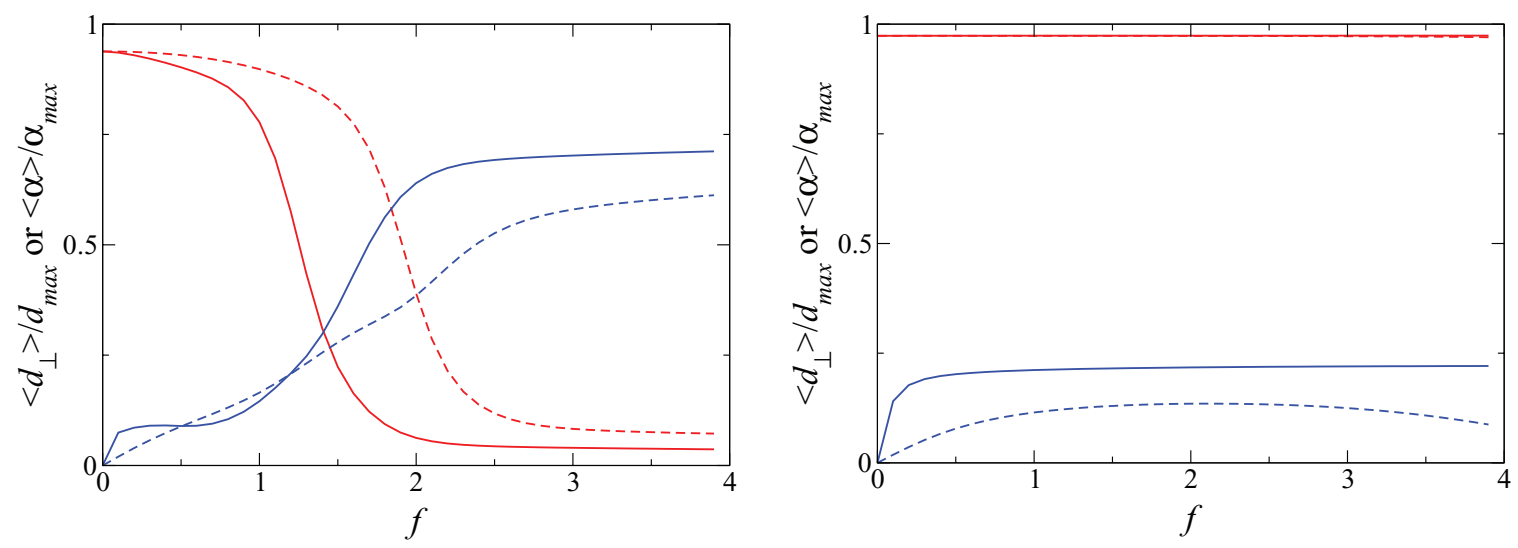

FIG. 7. Mean projected separation, $\left\langle d_{\perp}\right\rangle$ (blue curves), and mean wrapping angle, $\langle\alpha\rangle$ (red curves), for unconstrained wrapping of elastic filament around two cylinders. Left panel: $\sigma=1$. Right panel: $\sigma=10$. Solid curves correspond to $\mu=10$ and dashed curves correspond to $\mu=1$. Note that the results have been normalized to maximum displacement, $d_{\max }=l^{\prime}+2 R$, or to maximum angle.

approximation to be good is $\beta F L_{p} \gg 1$ or, equivalently, $f \mu / 2$ $\gg 1$. For our parameters in the left panel of Fig. 5 (with $\mu=10$ ), we have $f \gg 0.2$. Thus, for instance, in the middle of the looped phase $f \simeq 0.5$, hence $f \mu / 2 \simeq 2.5$; from Sec. III, we note that $\beta F L_{p}=2$ is not sufficiently large for the Gaussian approximation to hold and so we deduce that for the looped phase shown in Fig. 5 the unapproximated Mathieu states are necessary for a quantitative calculation.

\section{B. Two cylinders: Unconstrained wrapping}

We now allow the $\alpha_{i}$ to be a dynamical or annealed variable as opposed to the quenched case of Sec. IV A. As noted in the beginning of Sec. IV (case II), the corresponding realization would be an elastic filament wrapped around two cylinders with a fixed arc-length separation $l^{\prime}$ between the two points of pinning and no other constraints. The length of elastic filament adhering to the cylinders is now variable and so its length outside the region between the two cylinders and the effective length between them must both be allowed to vary dynamically.

In Sec. IV A we gave an expression for the partition function, $Z\left(\alpha_{1}, \alpha_{2}, l\right)$, for fixed angles of wrapping and fixed effective length of elastic filament between the cylinders. Therefore the total partition function for dynamical wrapping is clearly given by

$$
\begin{aligned}
Z= & \int d \alpha_{1} d \alpha_{2} \int_{0}^{\min \left(l / R, \alpha_{1}\right)} d \alpha_{1}^{\prime} \\
& \times \int_{0}^{\min \left(l / R-\alpha_{1}^{\prime}, \alpha_{2}\right)} d \alpha_{2}^{\prime} Z\left(\alpha_{1}, \alpha_{2}, l=l^{\prime}-R \alpha_{1}^{\prime}-R \alpha_{2}^{\prime}\right),
\end{aligned}
$$

where the integration is over the four wrapping angles which take into account the various ways for the wrapping to occur; for the $i$ th cylinder $\alpha_{i}$ (or $\alpha_{i}^{\prime}$ ) is the total (or internal) wrapping angle of the elastic filament, where internal refers to the length between the two cylinders. The computations were done with $\alpha_{i}, \alpha_{i}^{\prime}>0$; although the choice of sign for the first cylinder wrapping is arbitrary, we force symmetric wrapping on the second cylinder. We find that the antisymmetric configuration gives similar results and so do not report on it in detail. $\left\langle d_{\perp}\right\rangle$ can now be calculated by averaging the expression in Eq. (42) over the four wrapping angles.

Because of the ability to pre-compute the various contributions to the partition function before any integration and/or summations are done, computation time is kept to a minimum. However, there is an increase of several orders of magnitude in the computing cost compared with the fixed wrapping case.

In the following computations, we chose $l^{\prime}=2 \pi R$ and $\alpha_{i}$ $<12 \pi$. This puts a reasonable limit on the maximum wrapping angles but it already has a significant outcome and the amount of computing resources required is kept reasonable.

In Fig. 7 we plot the average projected length $\left\langle d_{\perp}\right\rangle$ versus $f$ for $\sigma=1,10$ and $\mu=1,10$. As $f$ is increased an unwrapping transition is indicated by a rapid decrease in $\langle\alpha\rangle$ and a corresponding increase in $\left\langle d_{\perp}\right\rangle$. The results for $\sigma=1$ show a clear unwrapping transition (Fig. 7, left). When the unwrapping occurs, the distance between the cylinders increases as one would expect, whilst in the wrapped phase there appears to be a small but potentially interesting effect causing a decrease in the separation as the force increases. This can be seen by the bump in the $\mu=10$ line at small $f$. As the rigidity $\mu$ is decreased the unwrapping is less pronounced; it is now easier for the filament between the cylinders to fluctuate more strongly on average.

For the case of $\sigma=10$ (Fig. 7, right), there is no unwrapping transition for both values of $\mu$ over the range of forces indicated. One would expect this kind of outcome when one changes the binding energy $\sigma$. Higher binding energy prevents unwrapping, holding the cylinders tightly onto the filament. The distance between the cylinders does however slowly increase with increasing rigidity owing to the straightening of the elastic filament.

We also note, in this case, that, although $\alpha$ does not change (it stays maximally wound), the average projected length between the two cylinders decreases for large enough external force. This counters the fact the filament will tend to straighten with increased external tension. It does, however, lead to the conclusion that for large external tension there is an effective attraction between the cylinders that pulls 
them together. This conclusion is corroborated also by direct evaluation of the interaction free energy between the cylinders as discussed below.

\section{Free energy of the unwrapping transition}

To understand the phase transition between a free and wrapped cylinder, i.e., the phenomenon of unwrapping and desorption, we calculate the free energy of wrapped and unwrapped systems. We restrict ourselves to the case of fixed wrapping angle (case I). This can be trivially calculated from our theory as we already have calculated the partition function:

$$
\beta \Phi\left(\alpha_{1}, \alpha_{2}, l\right)=-\ln Z\left(\alpha_{1}, \alpha_{2}, l\right) .
$$

Since we normalize all energies by subtracting the groundstate energy and do not put in wavefunctions for the end points, we normalize the system to have $Z=1, \Phi=0$ for a filament with no wrapped cylinders. We consider the free energy of a system for the cases of a single wrapped cylinder with wrapping angle fixed to $\alpha=\pi$, and of the system of two wrapped cylinders in the symmetric and antisymmetric configurations with $\alpha_{1}=\pi$ and $\alpha_{2}= \pm \pi$, respectively. In Fig. 8 we show the free energy for these three cases. The left hand and right hand plots compare the single cylinder with the double cylinder for the inter-cylinder separation $l=2 \pi R$ and the limit of large $l$ for the symmetric and antisymmetric configurations, respectively. We use $\mu=10.0$ and $\sigma=4.5$. For $f=0$, the free energy of the two cylinder system is essentially double that of a single cylinder, as would be expected. As the force increases so does the free energy.

In the antisymmetric case, at low external force the wrapping of both cylinders is energetically favorable. As the external tension increases the two cylinders stay wrapped until both desorb simultaneously (the line crosses the $\mathrm{x}$ axis). As we would expect in the limit of large separation, the critical force for unwrapping is the same as that of a single cylinder, however as the separation decreases a larger force is required as they are bound together.

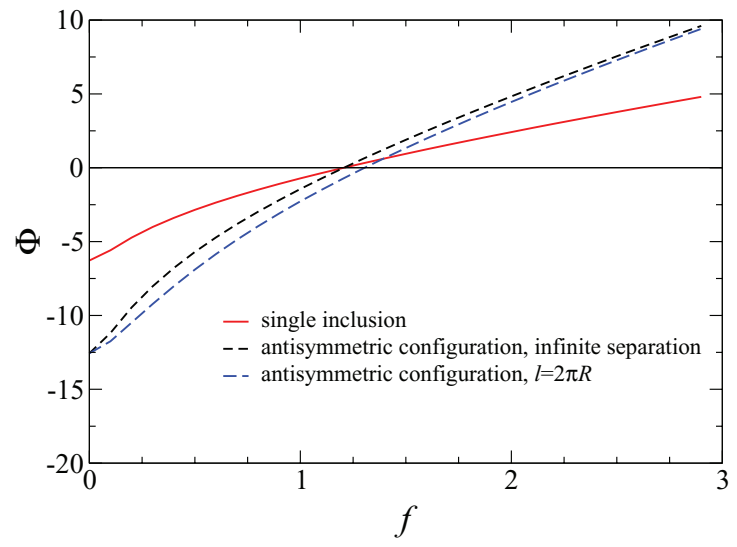

In the symmetric case we also see that at low external tension the double cylinder wrapping is energetically favorable. However, as the tension increases there comes a point where the single cylinder wrapping becomes energetically preferred and one of the cylinders then unwraps and leaves the chain. The details of this process cannot be captured appropriately in our model since we do not include the chemical potential for the unwrapped cylinders.

Increasing the external tension further unwraps the last cylinder. If the two cylinders are far apart we see the same behavior as for the antisymmetric case, as would indeed be expected. This agrees with previous studies showing that antisymmetric (symmetric) wrapping gives an attractive (repulsive) force. ${ }^{5}$

\section{EFFECTIVE INTERACTION BETWEEN TWO CYLINDERS}

We now consider the problem of effective interaction mediated by the elastic filament's fluctuations between two wrapped cylinders. The elastic and adhesive energy expressions are given as before.

\section{A. Constrained wrapping angles}

We first consider the case where the two cylinders are separated by fixed arc-length, $l$, and the wrapping angles are constrained at fixed values $\alpha_{1}$ and $\alpha_{2}$ (see the remarks identifying case I in Secs. IV and IV A). In numerical simulations we will assume furthermore that they are equal up to the sign, $\alpha_{1}= \pm \alpha_{2}$; the sign differentiates between the symmetric and the antisymmetric wrapping case. We can evaluate an effective interaction between the cylinders by fixing the (projected) horizontal separation $d_{\perp}$ between them and then calculating the corresponding free energy. The projected separation in the embedding space is defined as

$$
\begin{aligned}
d_{\perp}= & R \operatorname{sgn}\left(\alpha_{1}\right) \sin \left(\psi_{1}+\alpha_{1}\right) \\
& +\int_{s_{2}}^{s_{3}} d s \cos (\psi(s))-R \operatorname{sgn}\left(\alpha_{2}\right) \sin \left(\psi_{2}\right) .
\end{aligned}
$$

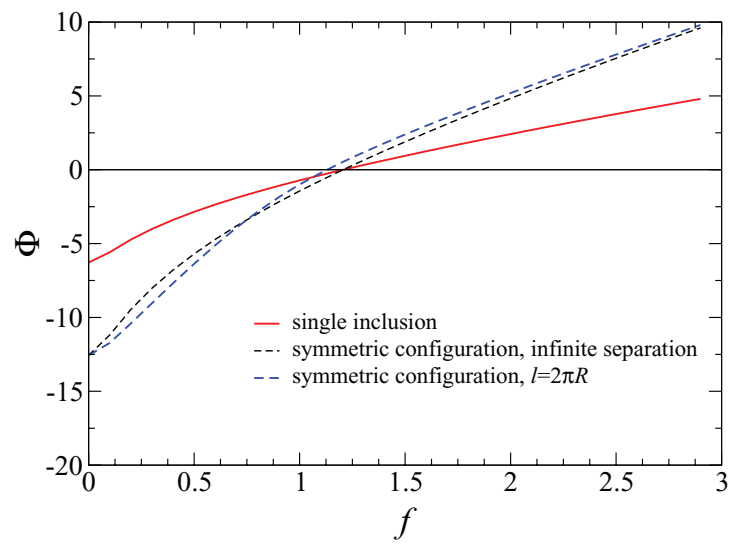

FIG. 8. Free energy as a function of the dimensionless external tension $f$ for $\mu=10$ and $\sigma=4.5$. Left: Red solid curve corresponds to a single cylinder $\alpha=\pi$, dashed curves correspond to antisymmetric double cylinder, $\alpha_{1}=-\alpha_{2}=\pi$. The lower (blue) curve corresponds to $l=2 \pi R$ and the upper (black) curve is limit of increased separation. Right: Red solid curve corresponds to a single cylinder $\alpha=\pi$, dashed curves correspond to symmetric double cylinder, $\alpha_{1}=\alpha_{2}=\pi$. The upper (blue) curve corresponds to $l=2 \pi R$ and the lower (black) curve is limit of increased separation. Note the double cylinder (black) curve that coincides with the single cylinder (red) curve at free energy equal to zero gives the limit of infinite separation. 
This constraint can be handled most conveniently by introducing an additional term into the total energy of the system via a Lagrange multiplier of the form $\lambda d_{\perp}$, where $\lambda$ can be interpreted as the force used to impose the constraint of fixed projected distance between the two cylinders in the horizontal direction.

The wrapping constraints can be implemented as before and one finds the appropriate partition function to be

$$
\begin{aligned}
Z_{\lambda}\left(\alpha_{1}, \alpha_{2}, l\right)= & \int d \psi_{1} d \psi_{2}\langle 0| \hat{O}_{\lambda}\left(\psi_{1}, \psi_{1}+\alpha_{1}\right) \\
& \times e^{-H_{\lambda} l / R \mu} \hat{O}_{\lambda}\left(\psi_{2}, \psi_{2}+\alpha_{2}\right)|0\rangle,
\end{aligned}
$$

where

$$
\begin{aligned}
H_{\lambda} \Psi_{\lambda m}(\psi) & =\left(-\frac{d^{2}}{d \psi^{2}}-\mu(f-\lambda) \cos (\psi)\right) \Psi_{\lambda m}(\psi) \\
& =\mu \varepsilon_{\lambda m} \Psi_{\lambda m}(\psi)
\end{aligned}
$$

with

$$
\begin{aligned}
& \hat{O}_{\lambda}\left(\psi_{1}, \psi_{1}+\alpha_{1}\right) \\
& \quad=\hat{O}\left(\psi_{1}, \psi_{1}+\alpha_{1}\right) \exp \left(-\beta \lambda R \operatorname{sgn}\left(\alpha_{1}\right) \sin \left(\psi_{1}+\alpha_{1}\right)\right)
\end{aligned}
$$

and

$$
\begin{aligned}
& \hat{O}_{\lambda}\left(\psi_{2}, \psi_{2}+\alpha_{2}\right) \\
& \quad=\hat{O}\left(\psi_{2}, \psi_{2}+\alpha_{2}\right) \exp \left(\beta \lambda R \operatorname{sgn}\left(\alpha_{2}\right) \sin \left(\psi_{2}\right)\right) .
\end{aligned}
$$

With this constraint the partition function $Z\left(d_{\perp}\right)$ becomes

$$
Z\left(d_{\perp}, \alpha_{1}, \alpha_{2}, l\right)=\int_{C-i \infty}^{C+i \infty} \frac{\mathrm{d} \lambda}{2 \pi i} e^{\beta \lambda d_{\perp}} Z_{\lambda}\left(\alpha_{1}, \alpha_{2}, l\right) .
$$

The appropriate constrained free energy then follows as:

$$
\beta \Omega\left(d_{\perp}, \alpha_{1}, \alpha_{2}, l\right)=-\ln Z\left(d_{\perp}, \alpha_{1}, \alpha_{2}, l\right) .
$$

The generalization to many wrapped cylinders is formally straightforward but computationally very tedious. One interesting future endeavour would be to assess the effects of non-pairwise additivity in the case of constrained and unconstrained wrapping around the interacting cylinders, i.e., the dependence of effective two-cylinder interaction on the presence of other wrapped cylinders along the elastic filament.

We can define related free energies appropriate for the sake of numerical calculation using

$$
\beta \Phi\left(\alpha_{1}, \alpha_{2}, l, \lambda\right)=-\ln Z_{\lambda}\left(\alpha_{1}, \alpha_{2}, l\right)
$$

and its Legendre transformation

$$
\Xi\left(l,\left\langle d_{\perp}\right\rangle, \alpha_{1}, \alpha_{2}\right)=\min _{\lambda}\left(\Phi\left(\alpha_{1}, \alpha_{2}, l, \lambda\right)-\lambda\left\langle d_{\perp}\right\rangle\right),
$$

which correspond to a system where either the polymer length, $l$, or the average horizontal displacement, $\left\langle d_{\perp}\right\rangle$, are fixed, respectively.

In Fig. 9 we plot $\Phi$ for $\lambda=0, \alpha_{1}=5 \pi / 8$, and $\alpha_{2}= \pm \alpha_{1}$ corresponding to symmetric and antisymmetric configurations, respectively. The length of elastic filament connecting the cylinders, $l$, is not a dynamical variable in our current model. However, by repeating the simulation for a sufficiently large range of $l$ we are able to produce the curves for fixed external force $f=0.4,1.0,2.0,3.0$ (from bottom to top). We assume that because there are pinning sites distributed along the filament, the cylinders can move through a tunnelling or hopping mechanism between sites and so $l$ and hence $\left\langle d_{\perp}\right\rangle(l)$ will vary to minimize the free energy $\Phi$. Thus we can infer from $\Phi$ the effective interaction between two cylinders in a system where $l$ is a dynamical variable. We choose to plot $\Phi$ versus the value of $\left\langle d_{\perp}\right\rangle(l)$, the projected horizontal separation between the two cylinders, since this is the more relevant observable. One can see the looped phase and the extended phase, corresponding to negative and positive $\left\langle d_{\perp}\right\rangle$, respectively. In the extended phase $\Phi$ increases with the magnitude of the external force for both kinds of wrapping symmetry, as one would expect. The effective interaction between the cylinders does, however, depend crucially on the symmetry of wrapping. In the asymmetric case (Fig. 9, left panel) the effective interaction is attractive in the extended phase. In the symmetric case, however, (Fig. 9, right panel), we see that the effective interaction is repulsive in the extended phase but then changes sign in the looped phase. In the extended phase, therefore, the cylinders with symmetric (antisymmetric) wrapping will move towards smaller (larger)

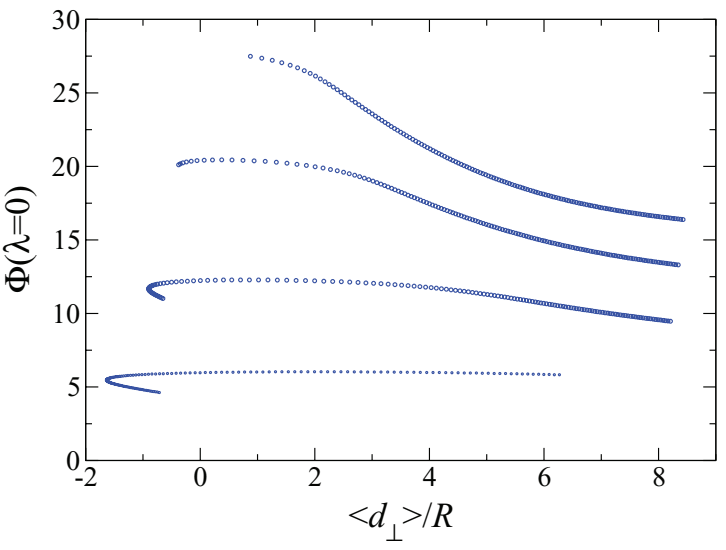

FIG. 9. Free energy as a function of the horizontal displacement projection $\left\langle d_{\perp}\right\rangle$ is shown for the case of constrained wrapping with $\alpha_{1}= \pm \alpha_{2}=5 \pi / 8$, $f=0.4,1,2,3$ (from lower to upper curve), $\mu=50$ and $\sigma=13.0$. In the antisymmetric case (left) the effective interaction is attractive and in the symmetric case (right) it is repulsive. 

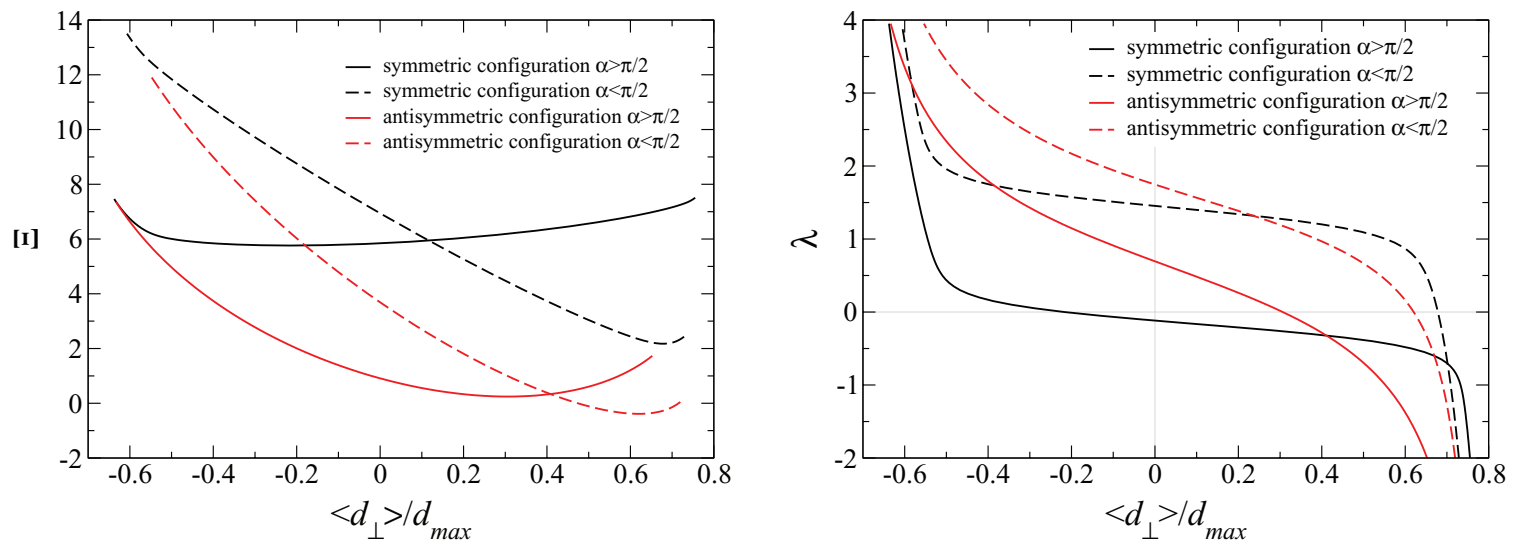

FIG. 10. Left: The free energy as a function of the horizontal displacement projection $\left\langle d_{\perp}\right\rangle$ (normalized to $d_{\max }=l+2 R$ ). Right: the required externally applied force, $\lambda$, as a function of the horizontal displacement projection $\left\langle d_{\perp}\right\rangle$. Here we consider the case of constant wrapping with $\alpha=5 \pi / 8>\pi / 2$ and $\alpha=3 \pi / 8<\pi / 2$ for $f=0.4, \mu=50, \sigma=13.0$, and $l=4 \pi R$.

$\left\langle d_{\perp}\right\rangle$; there is an effective attractive (repulsive) force between the cylinders. The higher the external force the bigger this effective force is between the wrapped cylinders.

In Fig. 10 we plot the free energy $\Xi\left(l,\left\langle d_{\perp}\right\rangle, \alpha_{1}, \alpha_{2}\right)$ defined in Eq. (54); $\Xi$ is a function of $\left\langle d_{\perp}\right\rangle$ at fixed $l$ (i.e., $l=4 \pi R$ in the figure). In the lefthand plot we show $\Xi$ for both symmetric and antisymmetric wrappings for $f=0.4, \mu$ $=50, \sigma=13.0$, and $\alpha=5 \pi / 8,3 \pi / 8$. The significance of the values chosen for $\alpha$ is that they are, respectively, $\gtrless \pi / 2$. For symmetric wrapping the equilibrium (minimum of $\Xi$ ) is in the looped phase for $\alpha<\pi / 2$ but it is in the extended phase for $\alpha>\pi / 2$. In contrast for antisymmetric wrapping, equilibrium is in the extended phase for both wrapping angles although at a smaller value of $\left\langle d_{\perp}\right\rangle(l)$ for $\alpha<\pi / 2$ than for $\alpha>\pi / 2$. In the right hand plot we show the projected separation $\left\langle d_{\perp}\right\rangle$ versus its conjugate variable $\lambda$. From Eq. (54) we note that $\lambda$ is determined as a function of $\left\langle d_{\perp}\right\rangle$ via

$$
\lambda=-\frac{\partial \Xi}{\partial\left\langle d_{\perp}\right\rangle}
$$

In an extended phase, $\left\langle d_{\perp}\right\rangle>0$, we have that $\lambda>0$ (or $\lambda$ $<0$ ) corresponds to an external force applied between the cylinders with magnitude $|\lambda|$ which pushes them together (or pulls them apart). In a looped phase, $\left\langle d_{\perp}\right\rangle<0$, we have that $\lambda>0$ (or $\lambda<0$ ) corresponds to an external force applied between the cylinders with magnitude $|\lambda|$ which pulls them apart (or pushes them together). We conclude that $\lambda\left\langle d_{\perp}\right\rangle>$ 0 (or $\lambda\left\langle d_{\perp}\right\rangle<0$ ) corresponds to an attractive (or repulsive) force between the cylinders.

These four choices for the variables $\left\langle d_{\perp}\right\rangle$ and $\lambda$ are shown separated by the dotted lines in the plot. The equilibria of the lefthand plot in Fig. 10 correspond to $\lambda=0$ in the righthand plot. Clearly, for symmetric wrapping and a wrapping angle $\alpha \sim \pi / 2$ the equilibrium value of the projected length is $\left\langle d_{\perp}\right\rangle$ $\sim 0$ giving a high probability for the cylinders to interact.

One should not forget here that in reality there may be other interactions between the wrapped cylinders (as in nucleosomes) that are not taken into account in this model: there would be direct electrostatic repulsions acting in real 3D space, as well as short range steric interactions when the cylinders are wrapped symmetrically, but not when they are wrapped antisymmetrically. It is the sum of all these complicated interactions that would need to be taken into account in a complete theory of cylinder wrapping.

\section{B. Unconstrained wrapping angles}

Next we consider the case of two cylinders with no constraints on the wrapping angles, corresponding to the second case (case II) as explained in the beginning of Secs. IV and IV B. This case is more complicated from the previous one as it entails an additional integration with respect to the two wrapping angles. The partition function can then be written in the same way as before in Eq. (44)

$$
\begin{aligned}
Z_{\lambda}\left(l^{\prime}\right)= & \int d \alpha_{1} d \alpha_{2} \int_{0}^{\min \left(l / R, \alpha_{1}\right)} d \alpha_{1}^{\prime} \\
& \times \int_{0}^{\min \left(l / R-\alpha_{1}^{\prime}, \alpha_{2}\right)} d \alpha_{2}^{\prime} Z_{\lambda}\left(\alpha_{1}, \alpha_{2}, l=l^{\prime}-R \alpha_{1}^{\prime}-R \alpha_{2}^{\prime}\right)
\end{aligned}
$$

and the variables are defined in Sec. IV B following Eq. (44). We consider the free energy, now for unconstrained wrapping angles, given by

$$
\beta \Phi\left(l^{\prime}, \lambda\right)=-\ln Z_{\lambda}\left(l^{\prime}\right) .
$$

and its Legendre transformation

$$
\Xi\left(l^{\prime},\left\langle d_{\perp}\right\rangle\right)=\min _{\lambda}\left(\Phi\left(l^{\prime}, \lambda\right)-\lambda\left\langle d_{\perp}\right\rangle\right),
$$

which again correspond to a system where either the polymer length, $l^{\prime}$, or the average horizontal displacement, $\left\langle d_{\perp}\right\rangle$, are fixed, respectively.

In the lefthand panel of Fig. 11 we plot the free energy, $\Phi$, of a quenched $l^{\prime}$ system (see remarks for case II in the beginning of Sec. IV) as a function of external force $f$ for a single cylinder and for two cylinders with symmetric wrapping and various arc-length separations. For antisymmetric wrapping the results are almost identical and we do not show them separately. We see that as $f \rightarrow 0$ and the arc-length separation $l^{\prime}$ becomes very large $\left(\left\langle d_{\perp}\right\rangle \rightarrow \infty\right)$, the free energy of two cylinders is twice the free energy of a single cylinder, 

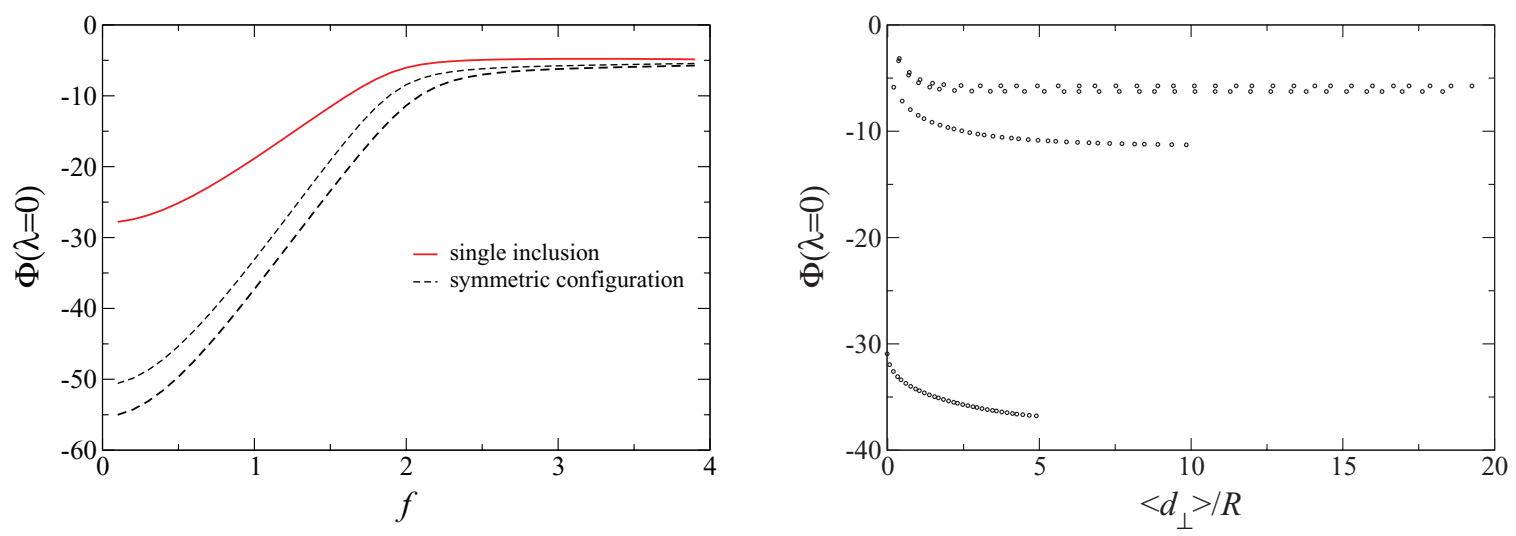

FIG. 11. The free energy for the case of unconstrained wrapping angles. Left: The free energy is shown as a function of external force $f$ for a single cylinder and for two cylinders with symmetric wrapping. The upper dashed curve is for $l^{\prime}=\pi R$ and the lower dashed curve corresponds to $l^{\prime}=20 \pi R$, being effectively infinite. As one expects, for large separation and low force, the free energy of two cylinders is double that of a single cylinder (red curve). Right: The free energy as a function of projected horizontal distance $d_{\perp}$, for $f=0.01,1,2,3$ (from bottom to top). The free energy decreases with separation (entropic effect) and increases with force. In both cases $\mu=1$ and $\sigma=1.25$.

which is a good consistency check. Note that for large $f$ the elastic filament can unwrap which allows the energetically favored small wrapping angle: The cylinders are pinned with no significant length of filament wrapped on them. This is in strong contrast to the case of constrained wrapping angle shown in Fig. 8 which shows a desorption transition due to the constraint imposed on the wrapping angle. In the righthand panel, we show the dependence of the unconstrained free energy, $\Phi$, on the projected separation $\left\langle d_{\perp}\right\rangle$. Although $l^{\prime}$ is a quenched variable, we are again able to produce these curve by repeating the numerical calculation for a sufficiently large range of $l^{\prime}$. The cylinders can move through a tunnelling or hopping mechanism between pinning sites and so $l^{\prime}$ and hence $\left\langle d_{\perp}\right\rangle\left(l^{\prime}\right)$ will vary to minimize the free energy $\Phi$. As the projected separation between the cylinders becomes smaller, i.e. they get closer together, we get an increase in the free energy corresponding to effective repulsive interactions which are not due to any hard-core repulsion between the cylinders, but are entropically generated. This entropy stems primarily from an "entropic wrapping" effect: By limiting the space between the cylinders they cannot wrap in as many ways as for large separations. Such entropic wrapping effects should be distinguished from the usual entropic configuration effects in semi-flexible polymers. In the latter the number of configurations of the polymer chain changes as we restrict the position of its ends and this leads to entropic polymer elasticity; for entropic wrapping effect the physical picture is altogether different.

In Fig. 12 we plot the conjugate variable $\lambda$ against the horizontal displacement $\left\langle d_{\perp}\right\rangle$ for a system with $l^{\prime}=2 \pi R$. Again $\lambda$ can be interpreted as an external force needed to maintain a mean projected separation $\left\langle d_{\perp}\right\rangle$, as described in Sec. V A. The response of the system to the external force is monotonic and attests to the fact that a large repulsive force, $\lambda>4$, greater than the external applied force, $f=3$ in this case, would be required to sustain a looped phase. The choice $l^{\prime}=2 \pi R$ is relatively short and we do not include the exclusion of one cylinder by the other which would restrict the possible configurations especially in a looped phase.
However, we treat the result here as an idealized case which is indicative of the possible likely configurations; in practice, the cylinders are not of infinite extent and so can be assumed to pass by each other more readily than the cylinders of this model. The absence of a looped phase in this idealized case is strong evidence that it is not likely to occur except for large $\lambda$ in a more realistic model of unconstrained wrapping. It should also be noted that the amount of computation time to explore all the parameter space in this case is considerable and so we are confined to investigating whether or not there is any significant non-trivial configuration that is likely to be realized.

We do not plot an analogue of the lefthand panel in Fig. 10 as it does not provide any extra information, however the free energy, $\Xi$, can be easily calculated numerically in our formalism.

From the numerical solution presented above it thus follows that the cylinder wrapping and the associated entropy presents yet another, apparently more important, source of polymer-mediated interactions between wrapped cylinders.

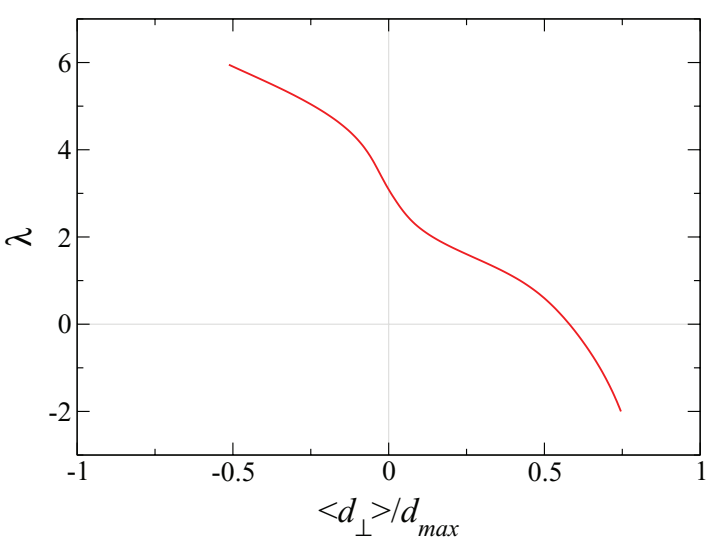

FIG. 12. The required externally applied force, $\lambda$, as a function of the horizontal displacement projection $\left\langle d_{\perp}\right\rangle$ (normalized to $d_{\max }=l+2 R$ ) is shown for the case of unconstrained wrapping with $f=3, \mu=1, \sigma=1.25$, and $l^{\prime}=2 \pi R$. 
Its source is the wrapping degrees of freedom that are constrained as the cylinders move closer together. To our knowledge, this source of effective interactions along a polymer chain has not before been clearly discussed in the literature.

\section{SUMMARY AND CONCLUSIONS}

We have analyzed wrapping equilibria of one and two cylinders on a semi-flexible filament driven solely by the elastic energy of the filament and the (adhesive) energy of wrapping around the cylinders. We derived the statistical properties and the free energy of wrapping in the case of one and two cylinders as well as the effective interaction free energy between two wrapped cylinders along the elastic filament. Our calculation is based on the functional integral representation of the partition function for the filament and is exact, within the confines of the worm-like chain model, the assumed form of the wrapping potential and the limit of a 2D Eulerian plane. We therefore neglect the effects of the local polymer twist between the wrapped particles ${ }^{35}$ as well as the orientational degrees of freedom of the wrapped particles themselves. ${ }^{36}$ The frozen orientational degrees of freedom assumed for the wrapped particles would be realistic in the small tension response whereas the model would have to be improved, relaxing the Eulerian plane constraint, in the case of sufficiently large tensions.

In Sec. II we presented the generalized theory for elastic filament wrapping on one or more cylindrical cylinders in one dimension. In Sec. III we calculated the wrapping transition for a single cylinder and showed that it is necessary to solve the full Schrödinger equation in order to obtain a good numerical value for the critical unwrapping external force. In Sec. IV we analyzed the exact solution for two cylinders pinned a fixed length apart on the elastic filament for both the looped phase, where the mean projected separation $\left\langle d_{\perp}\right\rangle$ is negative, and for the extended phase, where $\left\langle d_{\perp}\right\rangle$ is strictly positive and there are consequently no loops. The two cases considered are of constrained and unconstrained wrapping, respectively. In Sec. IV A the wrapping angles $\alpha_{1}$ and $\alpha_{2}$ on the respective cylinders are fixed: the case of constrained symmetric wrapping. This gives the most interesting results concerning the presence of a looped phase as discussed already by Rudnick and Bruinsma. ${ }^{5}$ We considered two values of wrapping angles: $\alpha_{1}=\alpha_{2}=\pi$ and $\alpha_{1}=\alpha_{2}=3 \pi / 8$. For the larger value there is a clear looped phase shown in Fig. 5 characterized by the average horizontal separation $\left\langle d_{\perp}\right\rangle<0$. The loop initially increases in size as the external tension $f$ is increased and eventually for sufficiently large $f$ the system switches over to the extended phase. In contrast, for smaller value of $\alpha_{i}$ shown in Fig. 6 there is no looped phase. In both cases the largest possible extension is close to its maximum possible value, $d_{\max }$ as determined by the arc-length of filament between the two cylinders and their radii, as one would expect. We conclude that a looped phase is possible for constrained symmetric wrapping and sufficiently large wrapping angles but is absent if the wrapping angle is too small. From Fig. 5 we see that where it does occur, the maximum loop size increases as the rigidity $\mu$ increases and is sustained for a range of tensions $f$; for $\mu=10$ the most negative values of $\left\langle d_{\perp}\right\rangle$ are approximately for $0.2<f<1$.

In Sec. IV B the two cylinders are pinned a distance $l^{\prime}$ apart along the contour of the elastic filament and the wrapping angles $\alpha_{i}$ are now dynamical (annealed) variables. In contrast to the constrained case the wrapping energy encoded in the dimensionless variable $\sigma$ plays a direct role in the values of the observables. The wrapping angle on a given cylinder is divided into two parts, which are the wrapping angles of the elastic filament wrapped to the left and to the right of the pinning point. In the two-cylinder case there are then four dynamical angle variables over which to sum, and this greatly increases the computer time required to carry out the calculation. We discussed in detail only the symmetric wrapping configuration as the antisymmetric one gives similar results. In Fig. 7 we show both $\left\langle d_{\perp}\right\rangle$ and $\langle\alpha\rangle$ (normalized to their maximum values) as a function of $f$ for $\sigma=1,10$ corresponding to small and large wrapping energy. For $\sigma=1$ there is a clear unwrapping transition for the two values of rigidity $\mu=1,10$ as the external tension $f$ increases, but for larger value of $\sigma$ the system remains maximally wound for all values of $f$. In particular, there is no looped phase indicated by $\left\langle d_{\perp}\right\rangle<0$; this is a characteristic of the unconstrained model. We chose separation $l^{\prime}=2 \pi R$ which is relatively short compared with the cylinder radius $R$ and treat the results as an idealized case which is indicative of the possible more realistic configurations. In practice, the cylinders are not cylindrical or of infinite lateral extent and so can be assumed to pass by each other more readily than the cylinders in the present model.

In Sec. IV C we calculate the free energy of the symmetric and antisymmetric constrained systems of two cylinders pinned a distance $l$ apart with wrapping angles $\alpha_{1}= \pm \alpha_{2}$ $=\pi$, respectively, and compare with the free energy of a single pinned cylinder with $\alpha=\pi$. In all cases the free energy is normalized by subtracting that of the elastic filament with no pinned cylinders. The results show that for large $l$ and for both the symmetric and antisymmetric configurations, we observe that desorption occurs for single as well as double cylinder systems simultaneously and for the same external tension. For small $l$ the situation is however different. For the antisymmetric configuration the double cylinder system is more strongly bound than for a single cylinder and remains bound after the single cylinder has been desorbed. In contrast, for the symmetric configuration, as the force increases, first the double cylinder becomes unstable leading to a single cylinder desorption, leaving a bound single cylinder which then desorbs as the external tension is increased further. The conclusion is that the symmetry of the double cylinder constrained wrapping has a crucial effect on the desorption transition. For unconstrained wrapping there is little structure since we do not associate a significant binding energy with the pinning site itself and so, as the external tension increases, the cylinders simply unwrap but remain pinned nevertheless. The effect in the constrained case is due to the competition between the wrapping and the entropic contributions to the free energy as a function of external traction and separation between the cylinders. 
In Sec. $\mathrm{V}$ we investigated the induced force between two pinned cylinders by introducing a force $\lambda$ conjugate to the projected distance $d_{\perp}$. We conclude that in the case of constrained wrapping the effective force, given by the slope of the curves, depends on the symmetry of configuration, being repulsive in the symmetric case and attractive in the antisymmetric case. The dependence of the effective force on the arclength separation $l$ between the cylinders follows closely the dependence on the projected separation between the cylinders in the direction of the external tension. In contrast, for the case of unconstrained wrapping we observe repulsion both for the symmetric as well as antisymmetric configurations. We interpret this repulsion as due to wrapping entropy that depends on the separation between the cylinders. This entropy differs from the usual polymer conformational entropy and one should distinguish between the two. The identification of the wrapping entropy presents a new concept in the analysis of the entropic effects in the context of polymer-particle complexes.

We finally calculate the force $\lambda$ required to sustain a given mean projected separation $\left\langle d_{\perp}\right\rangle$ and derived the free energy $\Xi\left(l,\left\langle d_{\perp}\right\rangle\right)$. For constrained wrapping we considered both symmetric and antisymmetric configurations with $\alpha_{1}= \pm \alpha_{2}=3 \pi / 8,5 \pi / 8$, respectively. In Fig. 10 we show $\Xi$ and $\lambda$ versus $\left\langle d_{\perp}\right\rangle / d_{\max }$. It should be noted that $\lambda\left\langle d_{\perp}\right\rangle>0(<0)$ corresponds to an intrinsic attractive (repulsive) force between the cylinders caused, e.g., by charges on the cylinders. The behavior of the $\lambda$ versus $\left\langle d_{\perp}\right\rangle / d_{\max }$ curves is consistent with this interpretation. We conclude that for the given choice of parameters the looped phase only occurs for symmetric wrapping and $\alpha_{1}=\alpha_{2}>\pi / 2$. The range of $\lambda$ chosen includes values where its magnitude exceeds the value of the applied external tension $f=0.4$. The response of $\left\langle d_{\perp}\right\rangle$ to $\lambda$ is as expected and we see a looped phase for a sufficiently repulsive intrinsic force. Other parameter choices can be investigated but we do not present the results here. In comparison, the case of unconstrained wrapping is basically featureless and the results are shown in Figs. 11 and 12, where only symmetric configuration is considered, the results for antisymmetric configuration being very similar. We note that the force between cylinders is repulsive in both these cases. We interpret this repulsion again as due to the wrapping entropy that depends on the separation between the cylinders. Since the wrapping entropy might also play an important role in the case of multiple wrapped cylinders and could promote very strong non-pairwise additive effects, we plan to study its effects very carefully in the future. Also we intend to introduce a chemical potential for exchange of the wrapped cylinders with a bulk phase in order to generalize the calculation of the distribution of nucleosomal core particles within the genomes. ${ }^{12,13}$

A major conclusion of our work is that for constrained wrapping, where the amount of elastic filament wrapped around the cylinder subtends a fixed angle at the center, there are two kinds of transition that can occur as a function of the dimensionless external tension $f$, rigidity $\mu$ and wrapping energy $\sigma$. For two or more wrapped cylinders there is a transition from a looped to an extended phase which is additionally affected by the direct inter-cylinder forces, and there are desorption transitions which are sensitive to the symmetry of the wrapping (determined by the relationship of the signs of wrapping angles) and also to the inter-cylinder separation.

A second major conclusion is that for unconstrained wrapping neither the looped phase nor the desorption transition are likely to exist. Instead, there is an unwrapping transition where amount of filament wrapped on each cylinders rapidly decreases as the external tension $f$ passes through a critical value. Correspondingly, the inter-cylinder distances rapidly increase from small to near maximum values within a very small tension interval.

\section{ACKNOWLEDGMENTS}

R.P. acknowledges support from Slovenian Research and Development Agency (ARRS) through Research Program P10055 and Research Project J1-4297. A.N. acknowledges support from the Royal Society, the Royal Academy of Engineering, and the British Academy through a Newton International Fellowship. We gratefully acknowledge support from Aspen Center for Physics, where this work was initiated during the workshop on New Perspectives in Strongly Correlated Electrostatics in Soft Matter (2010). We would also like to thank Martin M. Müller for introducing us to his work on the interaction between colloidal cylinders on membranes, which provided the initial inspiration for this work.

\section{APPENDIX A: CONSTRAINED WRAPPING EXPRESSED IN TERMS OF THE MATHIEU FUNCTIONS}

The path integral $K$ obeys the Schrödinger equation,

$$
\frac{\partial K\left(\psi, \psi^{\prime}, l\right)}{\partial l}=-H K,
$$

with boundary condition

$$
K\left(\psi, \psi^{\prime}, 0\right)=\delta\left(\psi-\psi^{\prime}\right) .
$$

This clearly means that $K\left(\psi+2 n \pi, \psi^{\prime}, l\right) \neq K\left(\psi, \psi^{\prime}, l\right)$ as it is violated at $l=0$ in the initial conditions. However, the propagator $K_{M}$ derived using the Mathieu functions, $\Psi_{n}$,

$$
K_{M}\left(\psi, \psi^{\prime}, L\right)=\sum_{n} \exp \left(-E_{n} l\right) \Psi_{n}(\psi) \Psi_{n}\left(\psi^{\prime}\right)
$$

has initial conditions

$$
K_{M}\left(\psi, \psi^{\prime}, 0\right)=\sum_{n} \delta\left(\psi-\psi^{\prime}-2 n \pi\right),
$$

and is clearly periodic. We can thus write

$$
K_{M}\left(\psi, \psi^{\prime}, l\right)=\sum_{n} K\left(\psi, \psi^{\prime}+2 n \pi, l\right) .
$$

If we take a single cylinder with fixed $\alpha$ (this is crucial in the argument that follows) we have

$$
\begin{aligned}
Z(\alpha)= & \int d \psi_{0} d \psi_{1} d \psi_{3} K\left(\psi_{0}, \psi_{1}, l_{1}\right) S\left(\alpha, \psi_{1}\right) \\
& \times K\left(\psi_{1}+\alpha, \psi_{3}, L-l_{1}-R|\alpha|\right),
\end{aligned}
$$

where $S$ is a general boundary term which is periodic in $\psi_{1}$. The initial integral over $\psi_{0}$ can clearly be taken over the interval $[0,2 \pi]$. If we restrict the integrals over $\psi_{1}$ and $\psi_{2}$ to 
$[0,2 \pi]$ and add on their integer changes by hand we get

$$
\begin{aligned}
Z(\alpha)= & \int_{0}^{2 \pi} d \psi_{0} d \psi_{1} d \psi_{3} \\
& \times \sum_{m, n} K\left(\psi_{0}, \psi_{1}+2 n \pi, l_{1}\right) S\left(\alpha, \psi_{1}+2 n \pi\right) \\
& \times K\left(\psi_{1}+2 n \pi+\alpha, \psi_{3}+2 m \pi, L-l_{1}-R|\alpha|\right),
\end{aligned}
$$

where we explicitly show that we restrict the integrals to $[0,2 \pi]$. Using the fact that $S$ is periodic for fixed $\alpha$ we finally obtain

$$
\begin{aligned}
Z(\alpha)= & \int_{0}^{2 \pi} d \psi_{0} d \psi_{1} d \psi_{3} \sum_{m, n} K\left(\psi_{0}, \psi_{1}+2 n \pi, l_{1}\right) S\left(\alpha_{2}, \psi_{1}\right) \\
& \times K\left(\psi_{1}+2 n \pi+\alpha, \psi_{3}+2 m \pi, L-l_{1}-R|\alpha|\right),
\end{aligned}
$$

and the obvious relation that

$$
K\left(\psi+2 n \pi, \psi^{\prime}+2 m \pi\right)=K\left(\psi, \psi^{\prime}+2(m-n) \pi\right) .
$$

We then change the summation variable over $m$ to $m-n$ to obtain

$$
\begin{aligned}
Z(\alpha)= & \int_{0}^{2 \pi} d \psi_{0} d \psi_{1} d \psi_{3}\left(\sum_{n} K\left(\psi_{0}, \psi_{1}+2 n \pi, l_{1}\right)\right) S\left(\alpha, \psi_{1}\right) \\
& \times\left(\sum_{m} K\left(\psi_{1}+\alpha, \psi_{3}+2 m \pi, L-l_{1}-R|\alpha|\right)\right)
\end{aligned}
$$

which then gives

$$
\begin{aligned}
Z(\alpha)= & \int_{0}^{2 \pi} d \psi_{0} d \psi_{1} d \psi_{3} K_{M}\left(\psi_{0}, \psi_{1}\right) S\left(\alpha_{2}, \psi_{1}\right) \\
& \times K_{M}\left(\psi_{1}+\alpha, \psi_{3}, L-l_{1}-R|\alpha|\right),
\end{aligned}
$$

which is the desired result expressed in terms of periodic Mathieu functions. The proof above for fixed wrapping angles can easily be extended to several cylinders with fixed wrapping angles.

\section{APPENDIX B: HORIZONTAL DISTANCE BETWEEN THE CYLINDERS}

From Eq. (42) we can write for the horizontal distance between the cylinders

$$
d_{\perp}=\left\langle d_{\perp}^{l}\right\rangle+R \operatorname{sgn}\left(\alpha_{1}\right)\left\langle\sin \left(\psi_{2}\right)\right\rangle-R \operatorname{sgn}\left(\alpha_{2}\right)\left\langle\sin \left(\psi_{3}\right)\right\rangle,
$$

where

$$
\begin{aligned}
\left\langle d_{\perp}^{l}\right\rangle= & \frac{1}{Z\left(\alpha_{1}, \alpha_{2}, l\right)} \int d s \int d \psi_{1} d \psi_{2} \Psi_{0}\left(\psi_{1}\right) C_{\alpha_{1}}\left(\psi_{1}\right) \\
& \times \sum_{m, n} \exp \left(-\epsilon_{m} s / R\right) \exp \left(-\epsilon_{n}(l-s) / R\right) \\
& \times \int d\left(\sin \psi_{s}\right) \Psi_{m}\left(\psi_{s}\right) \Psi_{m}\left(\psi_{1}+\alpha_{1}\right) \Psi_{n}\left(\psi_{s}\right) \Psi_{n}\left(\psi_{2}\right) \\
& \times C_{\alpha_{2}}\left(\psi_{2}\right) \Psi_{0}\left(\psi_{2}+\alpha_{2}\right),
\end{aligned}
$$

where a state has been inserted, with angle $\psi_{s}$ at the point $s$ of the first cylinder. We propagate the solution up to this point, calculate the horizontal projection, and then propagate to the remaining cylinder. We now find that

$$
\begin{aligned}
\left\langle d_{\perp}^{l}\right\rangle= & \frac{1}{Z\left(\alpha_{1}, \alpha_{2}, l\right)} \int d \psi_{1} d \psi_{2} \Psi_{0}\left(\psi_{1}\right) \cdot C_{\alpha_{1}}\left(\psi_{1}\right) \\
& \times \sum_{m, n} \int d s \exp \left(-\epsilon_{m} s / R\right) \\
& \times \exp \left(-\epsilon_{n}(l-s) / R\right) D_{m n}\left(\psi_{1}+\alpha_{1}, \psi_{2}\right) \\
& \times C_{\alpha_{2}}\left(\psi_{2}\right) \Psi_{0}\left(\psi_{2}+\alpha_{2}\right),
\end{aligned}
$$

where

$D_{m n}\left(\psi_{1}+\alpha_{1}, \psi_{2}\right)=\int d\left(\sin \psi_{s}\right) P_{m}\left(\psi_{1}+\alpha_{1}, \psi_{s}\right) P_{n}\left(\psi_{s}, \psi_{2}\right)$

and $C_{\alpha}$ is defined in Eq. (39). By noting that after the angular integration only the exponential dependence on $s$ remains, we integrate $s$ over the range $0 \leq s \leq l$ to finally get

$$
\begin{aligned}
\left\langle d_{\perp}^{l}\right\rangle= & \frac{1}{Z\left(\alpha_{1}, \alpha_{2}, l\right)} \int d \psi_{1} d \psi_{2} \Psi_{0}\left(\psi_{1}\right) C_{\alpha_{1}}\left(\psi_{1}\right) \\
& \times\left[\sum_{n} l e^{-\epsilon_{n} l / R} D_{n n}\left(\psi_{1}+\alpha_{2}, \psi_{2}\right)\right. \\
& \left.+\sum_{n, m \neq n} \frac{e^{-\epsilon_{m} l / R}-e^{-\epsilon_{n} l / R}}{\epsilon_{n}-\epsilon_{m}} D_{n m}\left(\psi_{1}+\alpha_{2}, \psi_{2}\right)\right] \\
& \times C_{\alpha_{2}}\left(\psi_{2}\right) \Psi_{0}\left(\psi_{2}+\alpha_{2}\right) .
\end{aligned}
$$

Note that because $L \gg l$, the effect of the filament lengths outside the cylinder region is encoded in the ground state factors $\Psi_{0}\left(\psi_{1}\right)$ and $\Psi_{0}\left(\psi_{2}+\alpha_{2}\right)$. The average distance $\left\langle d_{\perp}\right\rangle$ is easily computed since the various components can be precomputed. In a typical computation we pre-compute the lowest 20-100 Mathieu eigenfunctions and eigenvalues by recasting the Schrödinger equation in Eq. (29) as a matrix eigenvalue problem by discretizing the angle coordinate on the range $[0,2 \pi]$, and using proprietary NAG routines. ${ }^{45}$ The computation of the partition function is then straightforward and can be done with modest computing resources.

${ }^{1}$ B. Alberts, A. Johnson, J. Lewis, M. Raff, K. Roberts and P. Walter, Molecular Biology of the Cell (Garland Science, New York, 2002).

${ }^{2}$ L. J. Sun, B. R. Peterson, and G. L. Verdine, Proc. Natl. Acad. Sci. U.S.A. 94, 4919 (1997).

${ }^{3}$ R. Schlief, Annu. Rev. Biochem. 61, 199 (1992).

${ }^{4}$ DNA-Protein Structural Interactions, edited by D. M. J. Lilley (IRL, Oxford, 1995).

${ }^{5}$ J. Rudnick and R. Bruinsma, Biophys. J. 76, 1725 (1999).

${ }^{6}$ H. Schiessel, J. Phys.: Condens. Matter 15, R699 (2003).

${ }^{7}$ H. Boroudjerdi, Y.-W. Kim, A. Naji, R. R. Netz, X. Schlagberger, and A. Serr, Phys. Rep. 416, 129 (2005).

${ }^{8}$ K. K. Kunze and R. R. Netz, Phys. Rev. Lett. 85, 4389 (2000).

${ }^{9}$ K. K. Kunze and R. R. Netz, Phys. Rev. E 66, 11918 (2002).

${ }^{10}$ D. J. Clark and T. Kimura, J. Mol. Biol. 211, 883 (1990).

${ }^{11}$ N. Kaplan et al., Nature (London) 458, 362 (2009).

${ }^{12}$ G. Chevereau, L. Palmeira, C. Thermes, A. Arneodo, and C. Vaillant, Phys. Rev. Lett. 103, 188103 (2009).

${ }^{13}$ A. Arneodo, C. Vaillant, B. Audit, F. Argoul, Y. d'Aubenton-Carafac, and C. Thermes, Phys. Rep. 498, 45 (2011). 
${ }^{14}$ M. Rief, H. Clausen-Schaumann, and H. Gaub, Nat. Struct. Biol. 6, 346 (1999).

${ }^{15}$ C. L. Woodcock, Curr. Opin. Struct. Biol. 16, 213 (2006).

${ }^{16} \mathrm{~K}$. van Holde and J. Zlatanova, Semin Cell Dev. Biol. 18, 651 (2007).

${ }^{17}$ T. D. Yager, C. T. McMurray, and K. E. van Holde, Biochemistry 28, 2271 (1989).

${ }^{18}$ H. Boroudjerdi and R. R. Netz, Europhys. Lett. 64, 413 (2003).

${ }^{19}$ H. Boroudjerdi and R. R. Netz, Europhys. Lett. 71, 1022 (2005).

${ }^{20}$ H. Boroudjerdi and R. R. Netz, J. Phys.: Condens. Matter 17, S1137 (2005).

${ }^{21}$ H. Boroudjerdi, A. Naji, and R. R. Netz, Eur. Phys. J. E 34, 72 (2011).

${ }^{22}$ F. Thoma, Th. Koller, and A. Klug, J. Cell Biol. 83, 403 (1979).

${ }^{23}$ C. L. Woodcock, S. A. Grigoryev, R. A. Horowitz, and N. Whitaker, Proc. Natl. Acad. Sci. U.S.A. 90, 9021 (1993).

${ }^{24}$ J. Bednar, R. A. Horowitz, S. A. Grigoryev, L. M. Carruthers, J. C. Hansen, A. J. Koster, and C. L. Woodcock, Proc. Natl. Acad. Sci. U.S.A. 95, 14173 (1998).

${ }^{25}$ R. A. Horowitz, D. A. Agard, J. W. Sedat, and C. L. Woodcock, J. Cell Biol. 125, 1 (1994).

${ }^{26}$ A. Worcel, S. Strogatz, and D. Riley, Proc. Natl. Acad. Sci. U.S.A. 78, 1461 (1981).

${ }^{27}$ G. Wedemann and J. Langowski, Biophys. J. 82, 2847 (2002).

${ }^{28}$ H. Schiessel, Europhys. Lett. 58, 140 (2002).

${ }^{29}$ T. Schalch, S. Duda, D. F. Sargent, and T. J. Richmond, Nature (London) 436, 138 (2005).

${ }^{30}$ P. J. J. Robinson, L. Fairall, Van A. T. Huynh, and D. Rhodes, Proc. Natl. Acad. Sci. U.S.A. 103, 6506 (2006).
${ }^{31}$ J. Langowski and D. W. Heermann, Semin Cell Dev. Biol. 18, 659 (2007).

${ }^{32}$ J. Langowski, Eur. Phys. J. E 19, 241 (2006).

${ }^{33}$ P. M. Diesinger, S. Kunkel, J. Langowski, and D. W. Heermann, Biophys. J. 99, 2995 (2010).

${ }^{34}$ M. Depken and H. Schiessel, Biophys. J. 96, 777 (2009).

${ }^{35}$ E. F. Koslover and A. J. Spakowitz, Phys. Rev. Lett. 102, 178102 (2009).

${ }^{36}$ B. Sudhanshu, S. Mihardja, E. F. Koslover, S. Mehraeen, C. Bustamante, and A. J. Spakowitz, Proc. Natl. Acad. Sci. U.S.A. 108, 1885 (2011).

${ }^{37}$ T. R. Weikl, Eur. Phys. J. E 12, 265 (2003).

${ }^{38}$ M. M. Müller, M. Deserno, and J. Guven, Phys. Rev. E 76, 011921 (2007).

${ }^{39} \mathrm{~A}$ more general treatment of the problem where small membrane fluctuations parallel to the cylinders are taken into account as well can be found in P. Gosselin, H. Mohrbach, and M. M. Müller, Phys. Rev. E 83, 051921 (2011).

${ }^{40}$ S. Mkrtchyan, C. Ing, and J. Z. Y. Chen, Phys. Rev. E 81, 011904 (2010).

${ }^{41}$ J. Yan and J. F. Marko, Phys. Rev. E 68, 011905 (2003).

${ }^{42}$ D. S. Dean, R. R. Horgan, A. Naji, and R. Podgornik, J. Chem. Phys. 130, 094504 (2009).

${ }^{43}$ D. S. Dean, R. R. Horgan, and D. Sentenac, J. Stat. Phys. 90, 899 (1998).

${ }^{44}$ Note that as well as having a hard-core interaction along the length of the filament there is an additional hard-core constraint coming from cylinders occupying different arc-lengths but sharing the same position in space. Thus the simple Tonks gas model along the filament will contain additional long-range and non-local interactions.

${ }^{45}$ The Numerical Algorithms Group Ltd. 